\title{
Informative Signal Analysis: Metrology of the Underwater Geomagnetic Singularities in Low-Density Ionic Solution (Sea Water)
}

\author{
Osvaldo Faggioni' ${ }^{1,2}$, Maurizio Soldani' ${ }^{2}$, Giacomo Cozzani ${ }^{3}$, Rodolfo Zunino ${ }^{1}$ \\ ${ }^{1}$ DITEN-SEALab-ACM Laboratory, Department of Electric, Electronic, Telecommunication Engineering and Naval Architecture, \\ University of Genoa, Genoa, Italy \\ ${ }^{2}$ Istituto Nazionale di Geofisica e Vulcanologia, Rome, Italy \\ ${ }^{3} \mathrm{CNR}$-Consiglio Nazionale delle Ricerche, ITD-Istituto per le Tecnologie Didattiche, Genoa, Italy \\ Email: osvaldo.faggioni@unige.it, osvaldo.faggioni@ingv.it
}

How to cite this paper: Faggioni, $\mathrm{O}$. Soldani, M., Cozzani, G. and Zunino, R. (2018) Informative Signal Analysis: Metrology of the Underwater Geomagnetic Singularities in Low-Density Ionic Solution (Sea Water). Journal of Signal and Information Processing, 9, 1-23.

https://doi.org/10.4236/jsip.2018.91001

Received: September 18, 2017

Accepted: December 25, 2017

Published: December 28, 2017

Copyright $\odot 2018$ by authors and Scientific Research Publishing Inc. This work is licensed under the Creative Commons Attribution International License (CC BY 4.0).

http://creativecommons.org/licenses/by/4.0/

\begin{abstract}
The paper tackles the problem of reading singularities of the geomagnetic field in noisy underwater (UW) environments. In particular, we propose a novel metrological approach to measuring low-amplitude geomagnetic signals in hard noisy magnetic environments. This research action was launched to develop a detection system for enforcing the peripheral security of military bases (harbors/coasts and landbases) and for asymmetric warfare. The concept underlying this theory is the spatial stability in the temporal variations of the geomagnetic field in the observation area. The paper presents the development and deployment of a self-informed measurement system, in which the signal acquired from each sensor-observation node-is compared with the signal acquired by the adjacent ones. The effectiveness of this procedure relates to the inter-node (sensor-to-sensor) distance, L; this quantity should, on one hand, correlate the noise and, on the other hand, decorrelate the target signal. The paper presents the results obtained, that demonstrate the ability of self-informed systems to read weak magnetic signals even in the presence of very high noise in low-density ionic solutions (i.e. sea water).
\end{abstract}

\section{Keywords}

Geomatic, Geophysical Signals Processing, Geomagnetic Metrology, Harbor Protection, Counterterrorism Mag Security System

\section{Introduction}

In the current scenario of international politics, the asymmetric threat represents 
the main issue in enforcing harbor/coast security. This fact shifts the attention of researchers involved in harbor underwater (UW) protection studies, from the detection of volumetric sources such as submarine boats to the detection of quasipunctiform sources (such as intruding divers). From an operational point of view, the problem therefore evolves from the numerical classification of remote, low-frequency targets to closer, higher-frequency ones.

Acoustic systems are very effective at detecting remote volumetric sources, but this approach exhibits crucial issues in terms of confidence and effectiveness when approaching discontinuities in density (such as sea bottom, docks and any natural/artificial topographic singularity). However, the proximity to sea floor (or docks) is the operating environment of divers; hence we focus our attention on systems operating in those scenarios.

Magnetic detection is the best choice in the related peripheral detection. However, high magnetic noise characterizes the physical environment of sea bottom in harbors, and low amplitude of target signals (like those associated to divers) makes the conventional magnetic approach ineffective for diver detection. The classic approach to magnetic detection is based on "field of measure" in either direct form (measure of $\mathrm{A}[\mathrm{nT}]$ ) or incremental form (measure in $\Delta \mathrm{A}$ $[\mathrm{nT}])$. This metrological approach cannot solve the diver-detection problem because the amplitude of the target signal is negligible as compared with the noise, and a large portion of the target wavelength lies within the noise band. The problem is amplified in frequency domain detection, especially in short series, due to window effects of causal functions [1]. As a result, the use of mag methods to detect small targets (in high noisy UW environments) has been abandoned since a long time (some decades).

It is worth noting that this is not a technological problem, since magnetometers are commercially available having sensitivity in excess of the requirements for our purposes. Instead, the scenario poses a phenomenological issue, as the high magnetic noise, the low amplitude of target signal, and the specific measurements performed in ionic solutions severely limit the informative capability of the target signal [2]. In other words, the frequency band of the magnetic signal generated by a diver (possibly affected by swimming speed) lies within the frequency band of the magnetic noise; this prevents the use of frequency filters to increase robustness in the identification of the target signal. The metrology of mag singularity shifts the focus from raw field measurements to "space stability" measures, and introduces the distinction between "local" and "diffuse" magnetic signals. This new parameter of classification can solve the problem of detecting small magnetic sources in noisy environments [3].

In the last five years, a set of coordinated research projects were started to enhance the magnetic approach, by the Italian Plan of Military Research (PNRM: CAIMaN - Coastal Anti Intruders Magnetometers Network; La.Ma. - Land/ Marine Magnetometric Detector for Self-informed Systems), and by the European Defence Agency (EDA: HaPS - Harbour and base Protection Systems - 
Royal SWE Navy, IT Navy, Royal NOR Navy, GER Navy).

As compared with those research actions, this paper presents the advances and experimental results obtained by applying a novel technique, that is, the measurement of magnetic local singularity based on self-informed metrology.

\section{Problem Setting}

The problem of measuring magnetic signals in marine UW noisy environment involves two distinct aspects:

1) The high complexity of the environmental magnetic field in harbors (Figure 1), which stems from the interference with natural phenomena (internal and external to the Earth) and from artificial time transients (Figure 2);

2) The stability in the space domain (SD) of the individual signals composing the magnetic field. The measurement of a high-frequency magnetic anomaly in the space domain calls for a higher spatial sampling frequency (ultimately requiring greater accuracy in the positioning of the sensor array). From a metro-

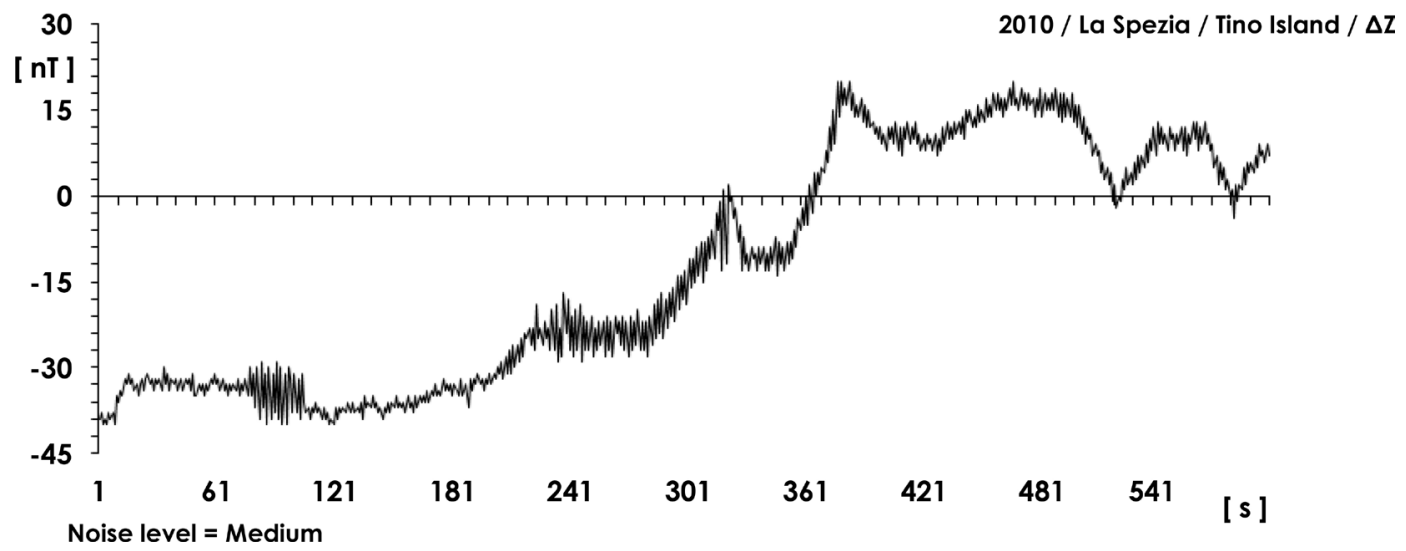

Figure 1. Island of Tino (La Spezia, Italy): example of UW magnetogram. Device: flux-gate magnetovariometer; component measured $\Delta Z$; device sensitivity 0.1 [nT]; graphic resolution 1 [nT] (by truncament); sampling rate $1[\mathrm{~Hz}]$; measurement period $T=10[\mathrm{~min}]$.

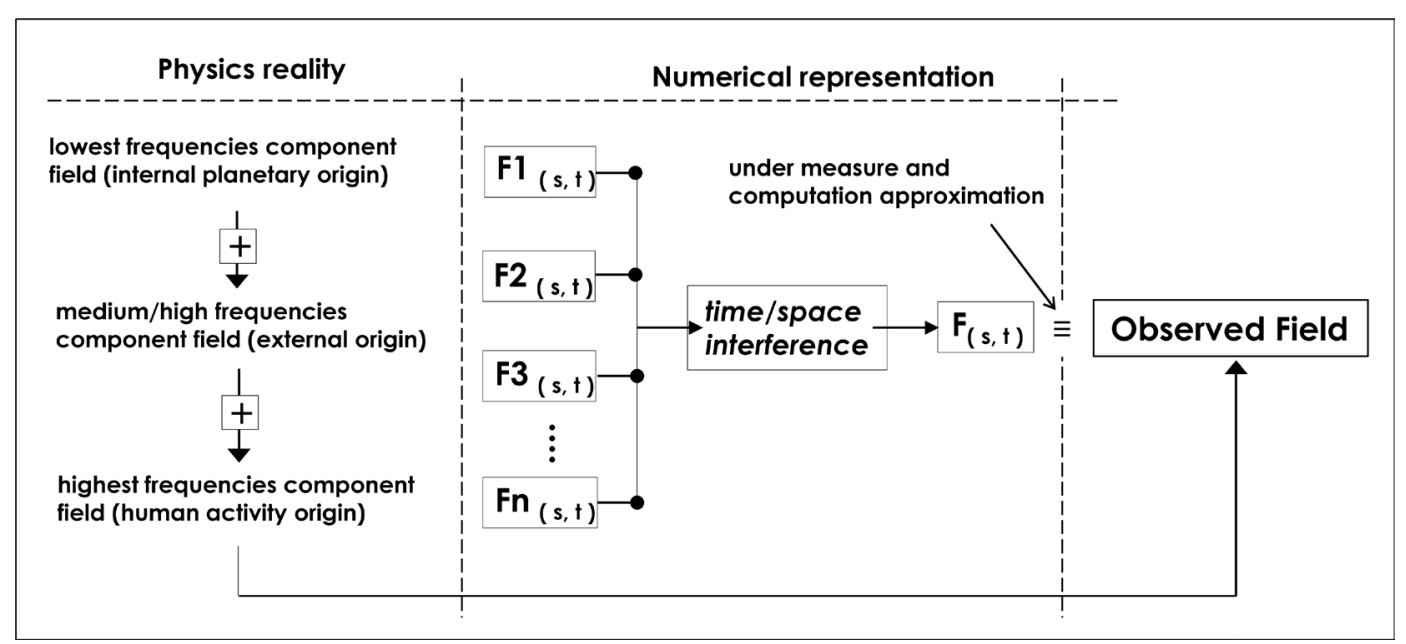

Figure 2. Numerical representation of the observed geomagnetic field (measured data) $F_{(s, t)} ; F 1_{(s, t)} \ldots, F n_{(s, t)}$ component fields of $F_{(s, t)}$ classified according to the frequency bands. 
logical point of view, this fact is related to the high-frequency SD stability, that is shorter than the low-frequency one (high spatial gradient $=$ short SD stability) [4].

This paper presents a metrological solution for both of the above issues. The method introduces a constraint parameter, $\mathrm{L}$, on the linear distance between the measurement points. As an effect of correctly setting $L$, noise signals on both magnetograms get correlated, while the target signal only shows up decorrelated in one magnetogram. The difference between the pair of magnetograms highlights the target signal.

The Earth's magnetic field (defined as the total measured field, or total field), $F$, embeds three families of basic component fields of different origins: internal planetary origin, extra-terrestrial origin, and human activity origin.

The internal geomagnetic field $\left(F_{i}\right)$, in turn, can be roughly classified in terms of a nuclear field, $F_{n}$, and s crustal field, $F_{c}$ The nature of the origin of $F_{n}$ is electromagnetic and mechanic (self-induction dynamo [5]), whereas $F_{c}$ is of ferromagnetic origin (it depends on ferromagnetic features of crustal rocks $\rightarrow$ Weiss. For our purposes, $F_{n}$ and $F_{c}$ exhibit long-period temporal variations (almost static), hence they are approximated as constant components of the total harbor magnetic field (background field parameter).

The extra-terrestrial field is of considerable interest for our purpose because it generates magnetic variations in a very wide spectral band. The high-frequency limit of this band (typical of D day's conditions) has the same order of amplitude (along the vertical component, $\mathrm{Fz}$ ) of the target magnetic signals associated with artificial sources, that are the aim of this research study [6]. These variations are classified starting from the geomagnetic planetary activity index, $K$, (three-hours horizontal parameter) [7].

The third component family $\left(F_{t}\right)$ is the actual core of our problem because the signals of our interest for detection belong to this family, and are convolved with all its components. We define this family of magnetic signals as $F_{h a}$ (human activity magnetic field) [8].

$$
F_{h a}=f e^{++/+++} F+e m f
$$

where:

- $F_{h a}$ is the human activity magnetic field;

- $f e^{+++++} F$ is the Weiss Domains activity from man-made objects;

- emf is the contribution of the magnetic induced field (very high activity for man-made metallic objects; these objects became sources of induced field when they are included in a time-transient magnetic space) [8].

According to the Konisberger factor $\left(K_{f}\right)$, emf produces an extremely interesting field in our activity: the band of induced fields $i F$;

$$
K_{f}=R / I
$$

where $R$ is the amplitude of the remanent magnetization and $I$ is the induced magnetization. The component of $F_{t}$ of interest involves component fields 
(temporal transients) that are characterized by frequencies of the same order of the target sources; let eff $F$ denote this band of effective field:

$$
\text { eff } F=D s t+D R+D P I+e m f+i F
$$

Although eff $F$ cannot be either effectively modeled or fully predicted, yet it is measurable [8] [9]. In numerical practice, the quantity eff $F$ (and also the total magnetic field $\left.F_{t}\right)$ is defined as the space-time field stemming from the interference of its elementary component fields: $F_{1 T}, F_{2 T}, \cdots, F_{n T}$ (Figure 2).

In fact, the ability to measure, from a standard metrological point of view, depends on the signal amplitude: the measurements acquired by sensors (i.e. magnetovariometers) can only describe effectively a signal that is evidently overmodulated in amplitude with respect to the UW magnetic environmental noise.

In diver detection applications, the target source is weak, quasi-punctiform, and cinematic, hence it is not measurable by a direct field measure, regardless of the specific sensitivity of the instrument.

This concept is formalized from the definitions of both informative contents, $Q$, of the observed field, and the informative capability or quality, $C_{i}$.

If we indicate by $E_{i}$ the energy associated with the $i$-th magnetic field elementary spectral component, the information content $Q$ is given by:

$$
Q=\sum_{i=0}^{N} E_{i}
$$

The information capability $C_{i}$ (defined as the capability to associate a single elementary spectral component with its physical generator) is given by the ratio of the energy $E_{i}$ to the total energy in which it is confused:

$$
C_{i}=E_{i} / Q
$$

The $C_{i}$ parameter lies in the range $0 \leq C_{i} \leq 1$ (where 0 corresponds to white noise or insufficient target signal amplitude, and 1 corresponds to monochromatic signals):

$$
\lim _{Q \rightarrow E_{i}} C_{i}=1 ; \lim _{Q \rightarrow \infty} C_{i}=0 ; \lim _{E_{i} \rightarrow 0} C_{i}=0
$$

The third limit indicates insufficient sensorial sensitivity (or absence of the magnetic energy) [8]. The time instability of the source frequency adds up to the above issues.

The target (UW equipment) space magnetic field $\left(F_{s}\right)$ depends on ferromagnetic phenomena and, at first-order approximation, is static.

The time measurement of the kinetic source is performed at a fixed location, hence the result is a $F_{t}$, whose spectral shape depends on target speed $(v)$ (Figure 3).

The variation range of a diver $v$ (not supported by DPV-Diver Propulsion Vehicle) lies in the range $100[\mathrm{~m} / \mathrm{h}]-2400[\mathrm{~m} / \mathrm{h}]$ [10] [11] (Figure 4). This indicates a spectral shift which virtually covers all high-frequency components of harbor magnetic noise (Figure 5).

This fact makes conjectural and ineffective any approach to implement detection by relying on frequency filters because (Figure 6): 
1) the target speed $v$ is unknown, hence the frequency range of its signal $F_{t}$ is also unknown;

2) it is not definable a non-conjectural cut frequency of the FFT filter for signal robustness.

\section{Measure of Singularity: The Self-Informing Protocol}

Space Stability (SS) is defined as the volumetric space occupied by the field produced by the magnetic source (e.g. diver). SS is a basic geometric parameter to classify the magnetic source type and its position from the associate magnetic field.

Singularity denotes the magnetic signal of the considered source when it is

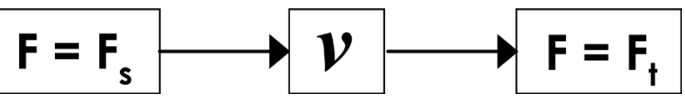

Figure 3. Effect of target speed $(v)$ and measurement system stability. Transduction of magnetic signal $F=F_{(s)}$ (static signal) by means of measurement action $\left(F=F_{(t)} \rightarrow\right.$ transient signal). Observed field $F=F_{(s, t)}$.

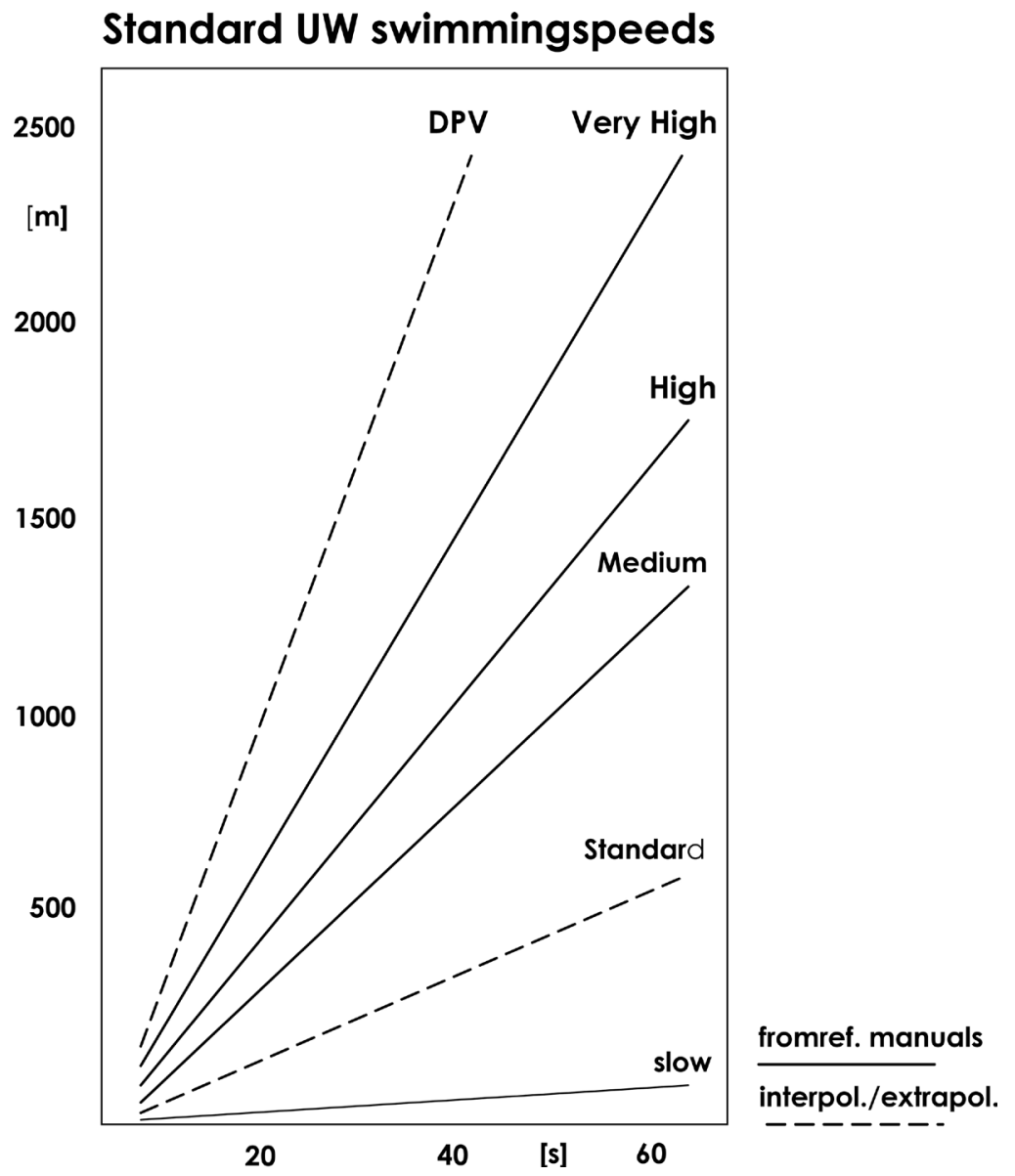

Figure 4. Table of uw swimming speed, (free or DPV—Diver Propulsion Vehicle power). From table of $\mathrm{O}_{2}$ consumption related to uw swimming speed. (Comando Subacquei Incursori, Italian Navy "Norme per le immersioni" [11] and US National Oceanic and Atmospheric Administration “Diving manual” [10]), Authors elaboration. 


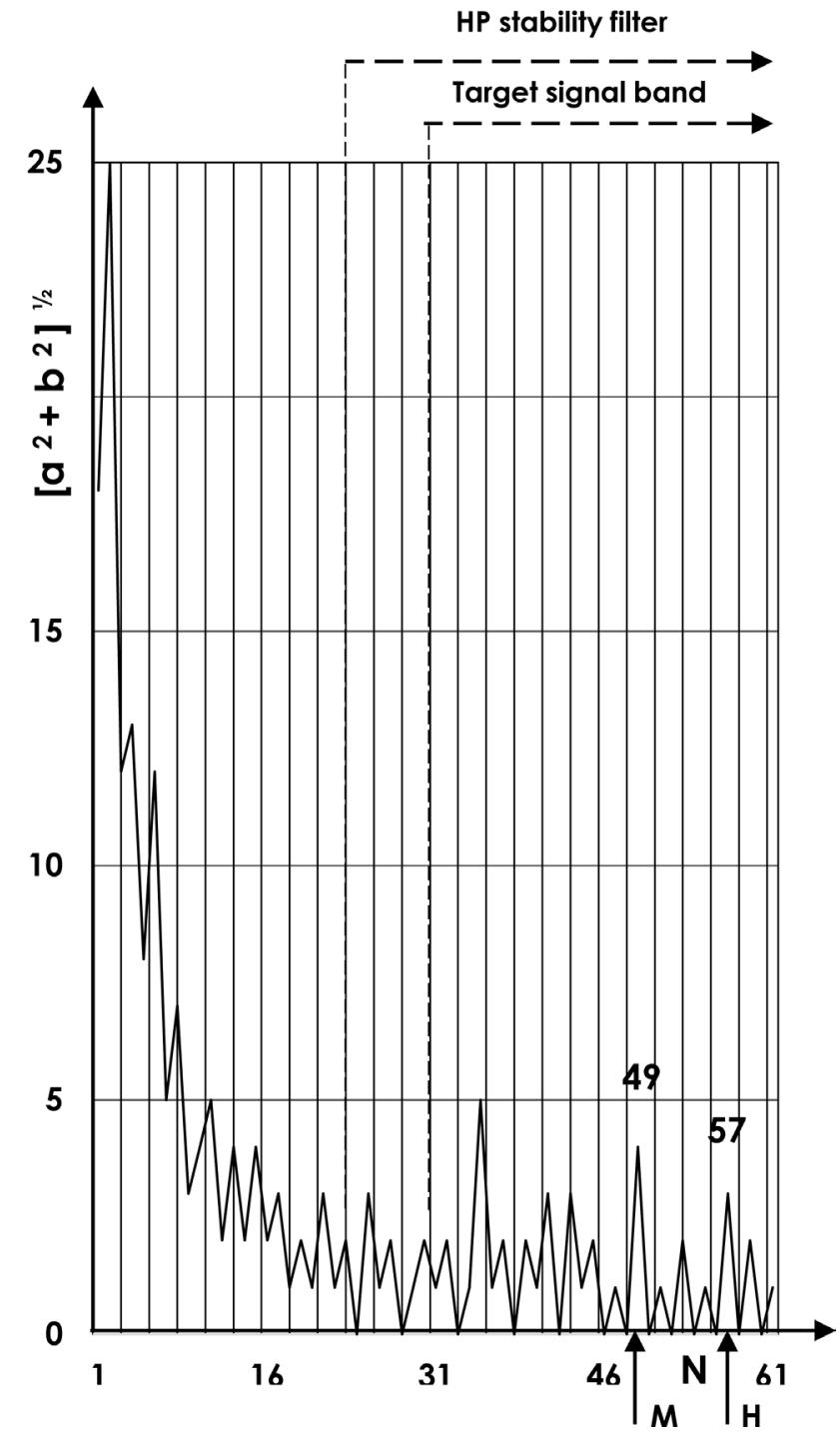

Figure 5. $\Delta \mathrm{Z}$ uw recording amplitude spectrum: $\mathrm{X}$ frequency order number, $\mathrm{Y}$ amplitude. $\mathrm{M} \rightarrow$ diver medium-low speed swimming (O.N. 49); H $\rightarrow$ high speed (O.N. 57). Target signal frequency band $31<$ O.N. $<61$, signal stability filter $\rightarrow$ HP FFT O.N. 23.

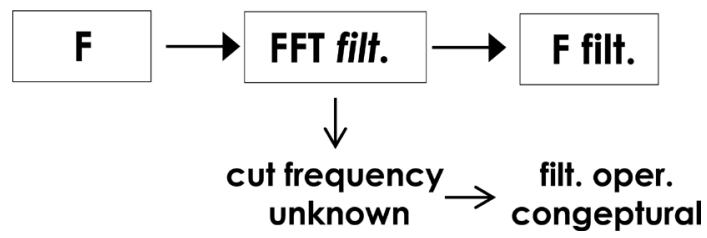

Figure 6. HP FFT robustness standard procedure of the target signal. The swimming speed is unknown, then the signal time frequency (measured) is not defined. This condition makes the filter response numerically correct but conjectural by physical point of view.

convoluted with other magnetic signals characterized by a wider spatial volume.

The aim is to describe formally such a singularity, regardless of the "temporal form" of its signal (due to the speed $v$ of the generating source), of its amplitude (except for the instrumental sensitivity), and of environmental noise (Figure 7). 


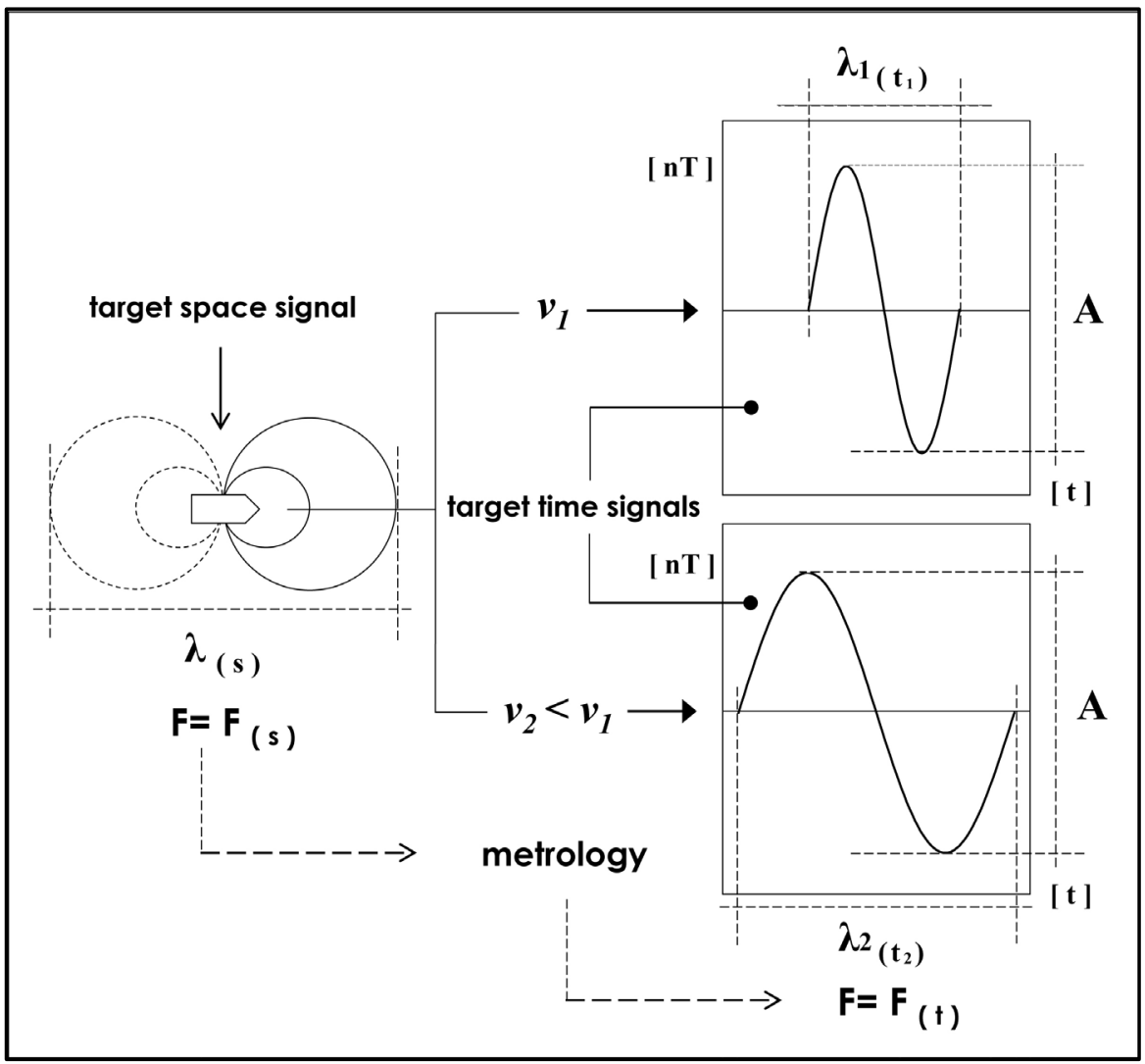

Figure 7. Variation of target signal's geometrical parameters (wavelength) related to the target speed.

The singularity measurement is based on the spatial stability of the perturbation that occupies a constant volume (at first approximation, this is a sphere having radius $r$ ).

This requires a measuring line (i.e., a chain of instruments) in which the observation nodes (sensors) are set at a distance, $L$, that is about $2 r$. This quantity is the largest distance to detect the source signal as a function of both its intensity and the observation resolution, and not of the target speed.

Therefore, the coverage of the geographical distances $(D)$ stems from a sequence of $n$ elementary sectors (length $=L$ ) where $n$ is given by $n=D / L+1$ [3] [12]. In experimental practice, linear underwater coverages with $D<100[\mathrm{~m}]$ have been realized so far [13].

The source is therefore detected only by one magnetovariometer, as a consequence of the transit of the target on its vertical line. In fact, this also holds in case of more intense signals, induced on the magnetometer that lies nearest to the transit location even if this does not coincide with the vertical of an instrument. An ambiguity only occurs when the transit happens halfway between two sensors, hence the signal is equally distributed on a pair of adjacent sensors [14]. With the exception of such condition, the two signals acquired by adjacent sensors only differ as a consequence of the presence of the diver signal. Therefore, the internodal distance $L$ decorrelates the target signal but maintains approx- 
imately correlated the noise.

The sequential comparison of consecutive sensors (Zero Control) informs the system about the singularity (target signal), and detects it from the deconvolution of the "noise" signal (geomagnetic underwater environment) from the "noise plus target" signal, regardless of the amplitude and of the frequency.

A further control (Ambiguity Control) can resolve the ambiguity by using the same system architecture: in the Ambiguity Control, every zero signal provided from the comparison of two adjacent sensors is compared (in deconvolution) with the signal acquired by the next-in-line sensor. An elementary cell of the selfinformed observation systems therefore holds three measuring points (Figure 8). Such a cell has length $2 L$ and covers a linear distance $d$ given by $d=3 L$.

This final control provides the certainty of absence of singularities (normal condition) or identifies unambiguously the presence of a singularity (alarm condition) within an elementary cell. The length to be protected is virtually divided into elementary cells that are partially overlapped, and the detection is performed by a chain of finite elements providing a detection localization equal to $L / 2[7]$.

\section{Measurements, Data, Results}

This section shows the results obtained by the self-informed detection system (vertical component $\Delta Z$ - Table 1 ) in real harbor testing conditions. The source

\section{Architecture of the elementary cell for selfinformed mag detection systems}

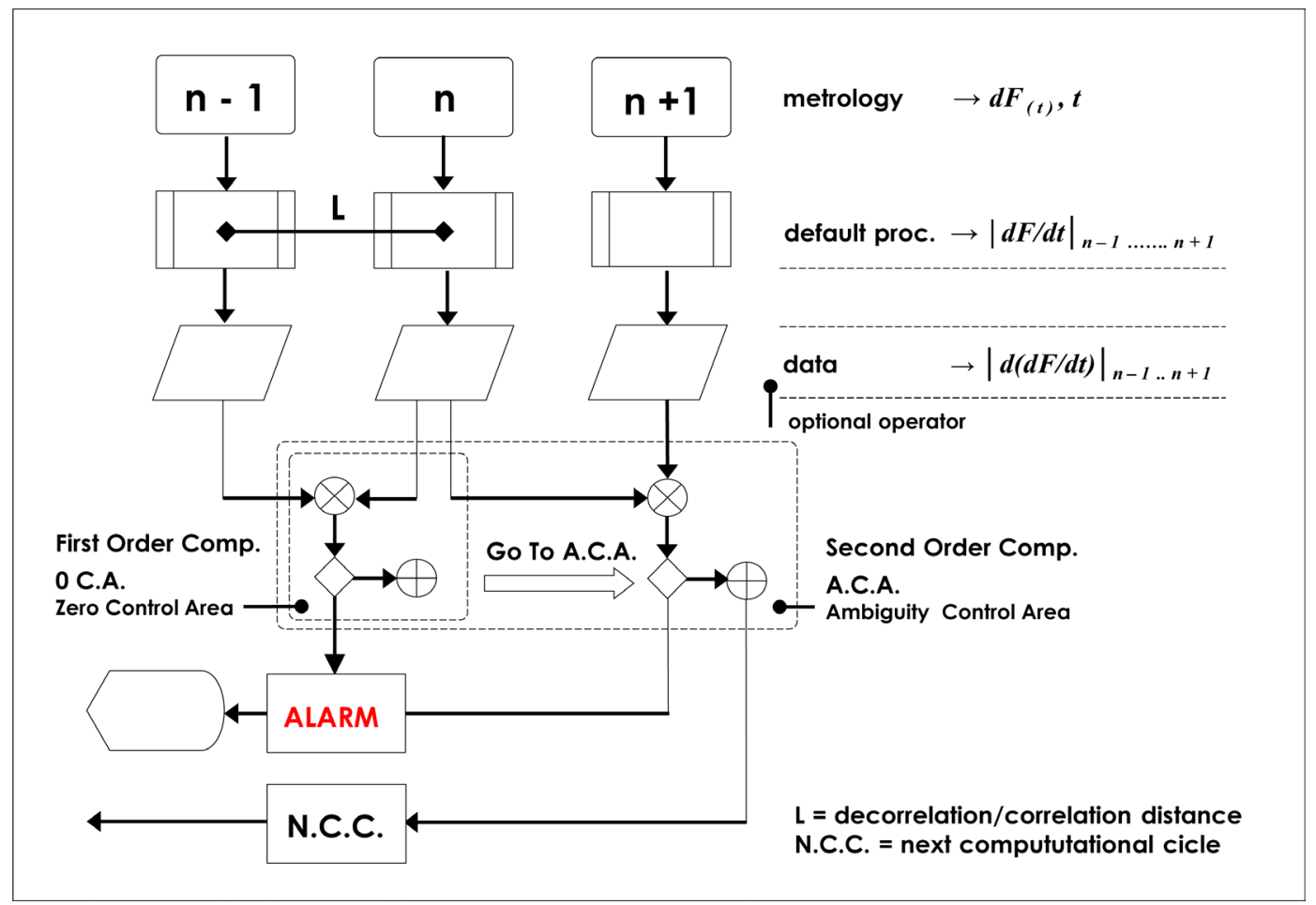

Figure 8. Self-information intelligence protocol: general plan and data processing flow of the "SUPERGUARD" elementary cell to measure and classify singularity signal. 
Table 1. Parameter of the measure and graphycal design of the magnetic recordings shown in this Paragraph 4.

\begin{tabular}{c}
\hline Measure and graphycal parameters \\
Measure \\
indipendent variable: time, unit $[\mathrm{s}]$, sampling rate $[\mathrm{s}] ;$ \\
dipendent variable: $\Delta \mathrm{F}$ measure, unit $1[\mathrm{nT}]$; records acquisition frequequency $1[\mathrm{~Hz}]$ \\
$\mathrm{Graphycs} \mathrm{elaborations}$ \\
$\mathrm{X} \rightarrow[\mathrm{s}], \mathrm{Y} \rightarrow[\mathrm{nT}]$, approx. $1[\mathrm{nT}]$ (truncated)
\end{tabular}

is provided by a diver; equipment includes commercial-class (single tank) devices, and measuring instruments are flux-gate magnetovariometers [15]. With reference to Figure 9 and Figure 10 we note the ineffectiveness of FFT filtering (standard approach) to detect the target signal. The magnetogram in Figure 9 shows a noise signal (A) whit the same wavelength order as the target signal (B): A and B signals are not classifiable in the spectral representation. In the case of Figure 10 (time domain signal interference (a)) the interference of target signal (D) with recurring noise (more or less a square windows) makes unstable the spectral computation and uncertain the FFT signal classification [16]. We present now the results performed by "singularity" approach in first-order processing conditions (0 C.A.) and in second-order ones (A.C.A.) (Figure 8).

Before applying the self-informed protocol, the signal was filtered by a HP FFT procedure (HP stability filter, Figure 5) to exclude the recording of lowfrequency signals that were certainly unrelated to the signal of a possible diver [9]; this bypassed the issue of the non-stationarity of sequence to be analyzed (Figure 11). This is a very insidious problem for short numerical series [15] [17]. The examples present the signals recorded in correspondence to a diver intrusion by the control magnetic barrier, which included six instruments connected to a control station (Master Station) and placed about five meters from the coast.

The divers involved in the trials were provided with commercial underwater equipment (self-breathing apparatus and single tank) and proceeded on approach routes that were oriented along Zero-Control and Ambiguity-Control reading conditions (Figure 12).

- Zero Control Area 0 C.A. (first order control) performance ( $1^{\text {st }}$ ex.)

The 0 C.A. protocol checked the condition of field variation between two successive instruments (to avoid the condition of "zero difference"), identified a local signal (singularity) on one of the two instruments, then alarmed the system (Figure 13).

The comparison between the recordings was performed according to the scheme shown in Figure 13, and was executed in the frequency domain (deconvolution). Instead, under conditions of system management from GPS clock (synchronous measures), the comparison was executed in the time domain (difference).

The graphs give the magnetograms acquired during an approach route of type 


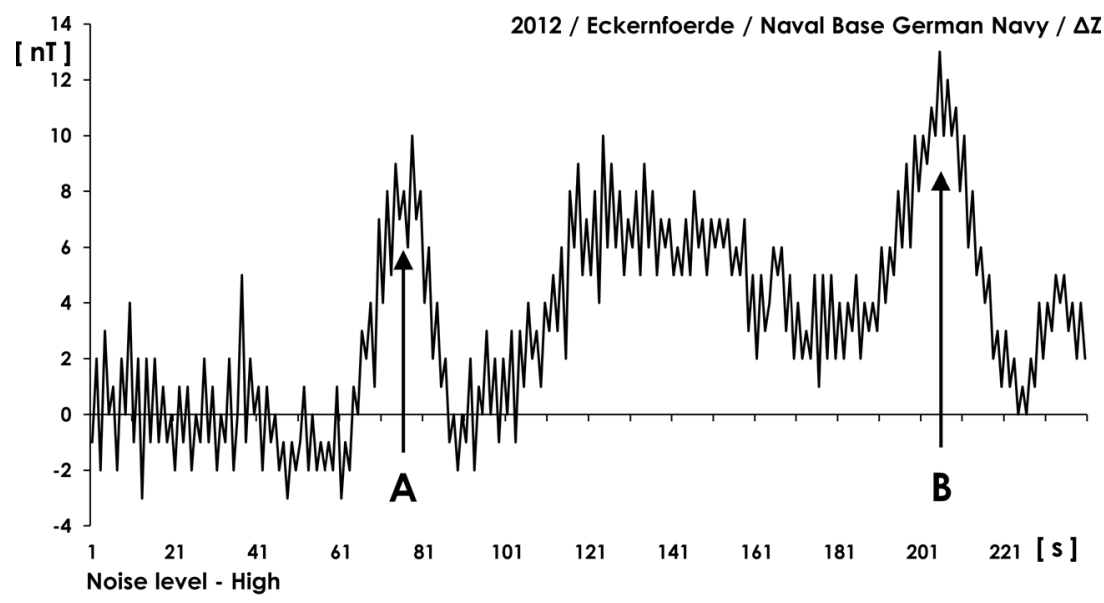

(a)

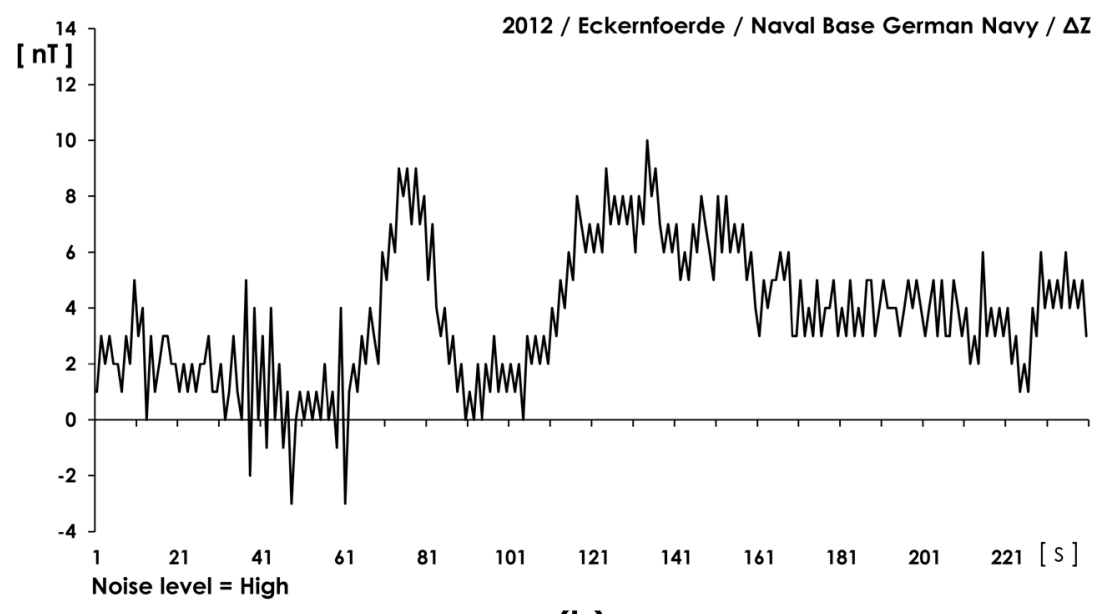

(b)

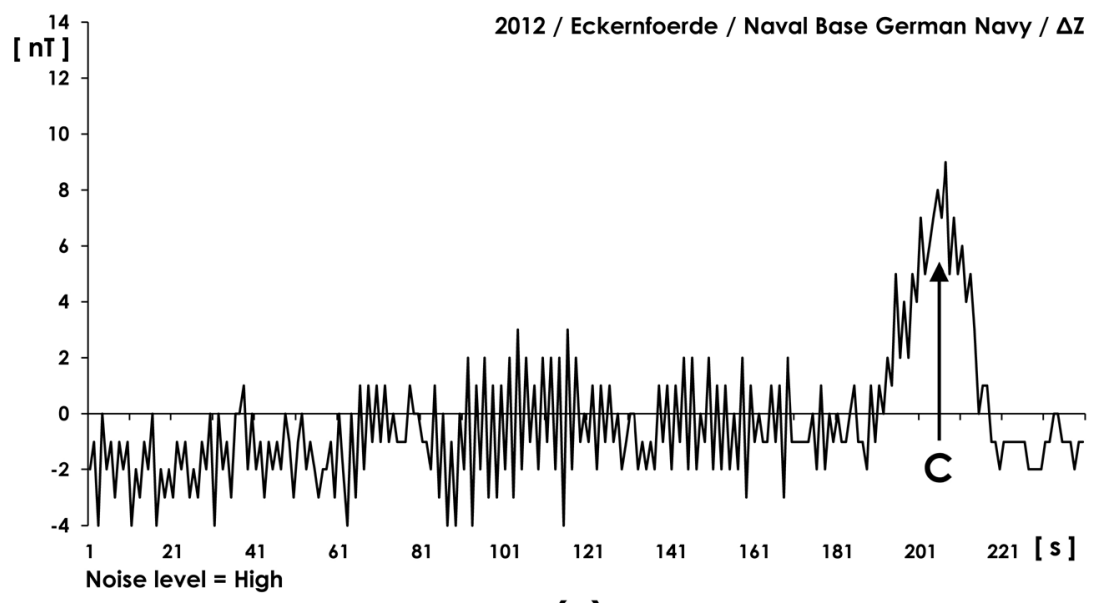

(c)

Figure 9. (a) Measured signal reflecting two phenomena: a diver that crosses the chain over sensor No. 2 (pulse "B"), and environmental noise (Pulse "A"). A direct observation cannot distinguish noise-generated pulses from target pulses, hence pulse " $\mathrm{A}$ " is not classifiable by standard frequency filtering approach (case of same wavelength and amplitude noise-target signal); (b) noise recording from sensor No. 1 (reference) during the same time interval of the diver-signal from sensor No. 2 (sentinel). The distance between the sensors correlates the noise and decorrelates the target signal; target speed $\mathrm{v}$ slow/medium, noise level was high, $X[\mathrm{~s}], Y[\mathrm{nT}]$, samplig rate $f z=1[\mathrm{~Hz}], T=4$ [min], measured at Eckernförde 2012; (c) devoconvolution result: sentinel magnetogram $\rightarrow$ reference magnetogram (under computation approximations; the result highlights target signal). 


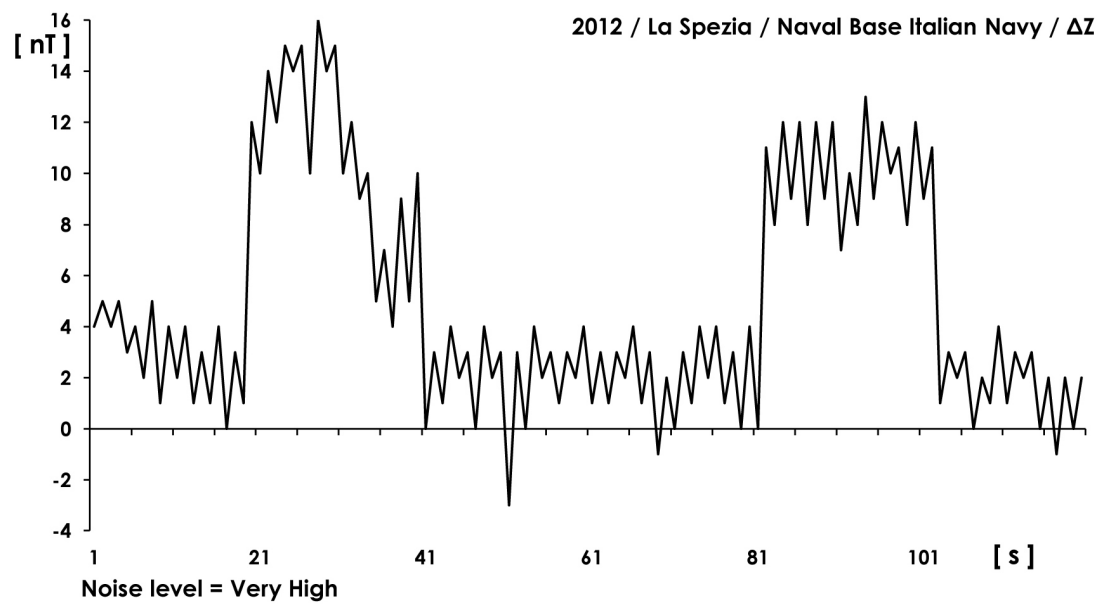

(a)

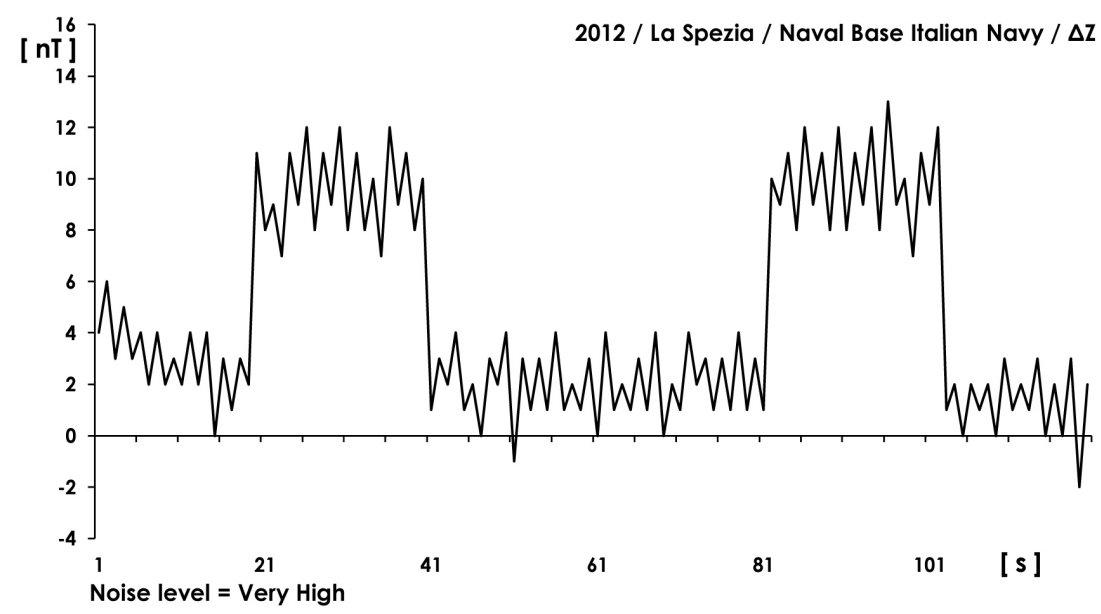

(b)

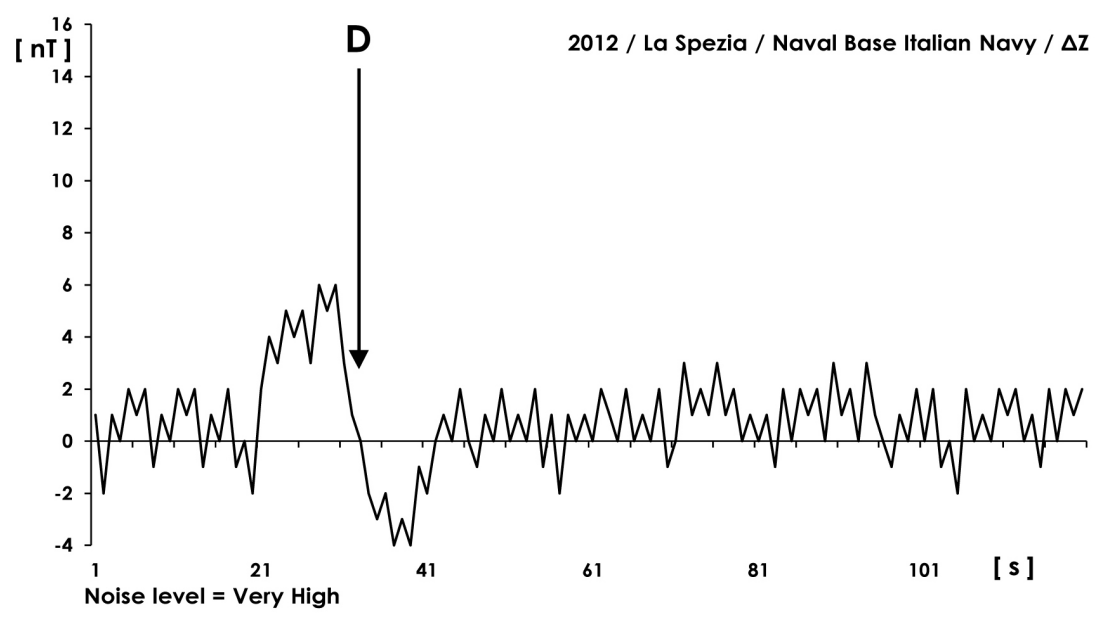

(c)

Figure 10. Superposition of the target signal (diver crossing the chain above sensor No. 3) over recurrent noise (human activity); $X[\mathrm{~s}], Y[\mathrm{nT}]$, samplig rate $f z=1[\mathrm{~Hz}], T=2[\mathrm{~min}]$, target speed: slow/medium, noise level: very high, La Spezia 2012; (a) measured overall signal at the sentinel station $(\Delta \varphi \cong 0)$, which shows the impossibility to discriminate diver-related from noise-generated events; (b) (noise) signal acquired by sensor No. 2 (reference); (c) signal worked out from the deconvolution a $\rightarrow$ b: target event D (under computation approximations). 


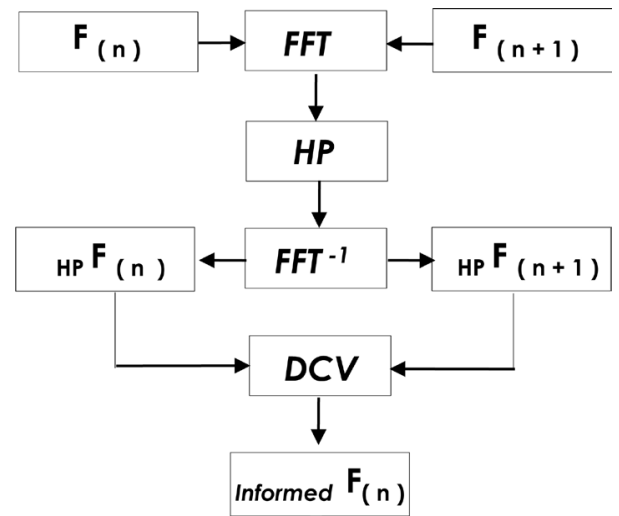

Figure 11. Target signal's robustness standard procedure. Computation flow of the HP FFT filtering to avoid low frequency bands (Figure 5) to make stationary the data series to be sent to singularity computation protocol.

1, followed by a diver from offshore to the coast (Figure 12, ex.1). The diver route crossed the self-informing observation chain almost at the perpendicular line of an observation node (magnetometer 2).

The distance between nodes ( $L$ in Figure 8 ) decorrelated the target signal (Figure 9(a)), recorded by the sentinel measure magnetometer 2 (Figure 12), from the background field acquired by magnetometer 1, which served as a reference (Figure 9(b)).

The comparison (either frequency deconvolution or time subtraction) between the reference (noise) signal at magnetometer 1 (Figure $9(\mathrm{~b})$ ) and the (diver signal + noise) signal at sensor 2 (Figure 9(a)) provides, as a residue, the signal generated from the diver moving near the measurement magnetometer (Figure 9(c)). This pinpoints the magnetic singularity present in the record of this magnetometer.

The singularity is defined as a $\Delta F$ magnetic field variation that is present only in the geomagnetic space volume controlled by a one magnetometer, the sentinel one, in this case the magnetometer 2; see Figure 12 (ex. 1).

The observation of Figure 9 (c) highlights as the high-frequency noise signals persist after the action of the protocol 0 C.A. and in most of the residual registrations, this noise is rather amplified.

The residue depends to the distance ( $L$ ) (between the observation nodes); it is decorrelating the magnetic target signal (diver) and is correlating the noise too; but at the same time it is too wide to correlate the high-frequency components of the noise. As a result, the target signal survives to the 0 C.A. filtering and the noise doesn't survive.

- Zero Control Area 0 C.A. (first order control) performance $\left(2^{\text {nd }}\right.$ ex.)

Figure 10(a) presents the action of 0 C.A. when a recurring artificial magnetic noise (that is not ascribable to a singularity) is superimposed to a typical magnetic singularity (signal associated to a diver that crosses the SUPERGUARD control barrier). The operative condition of the system 0 C.A. is the same than the previous example as per Figure 13. 


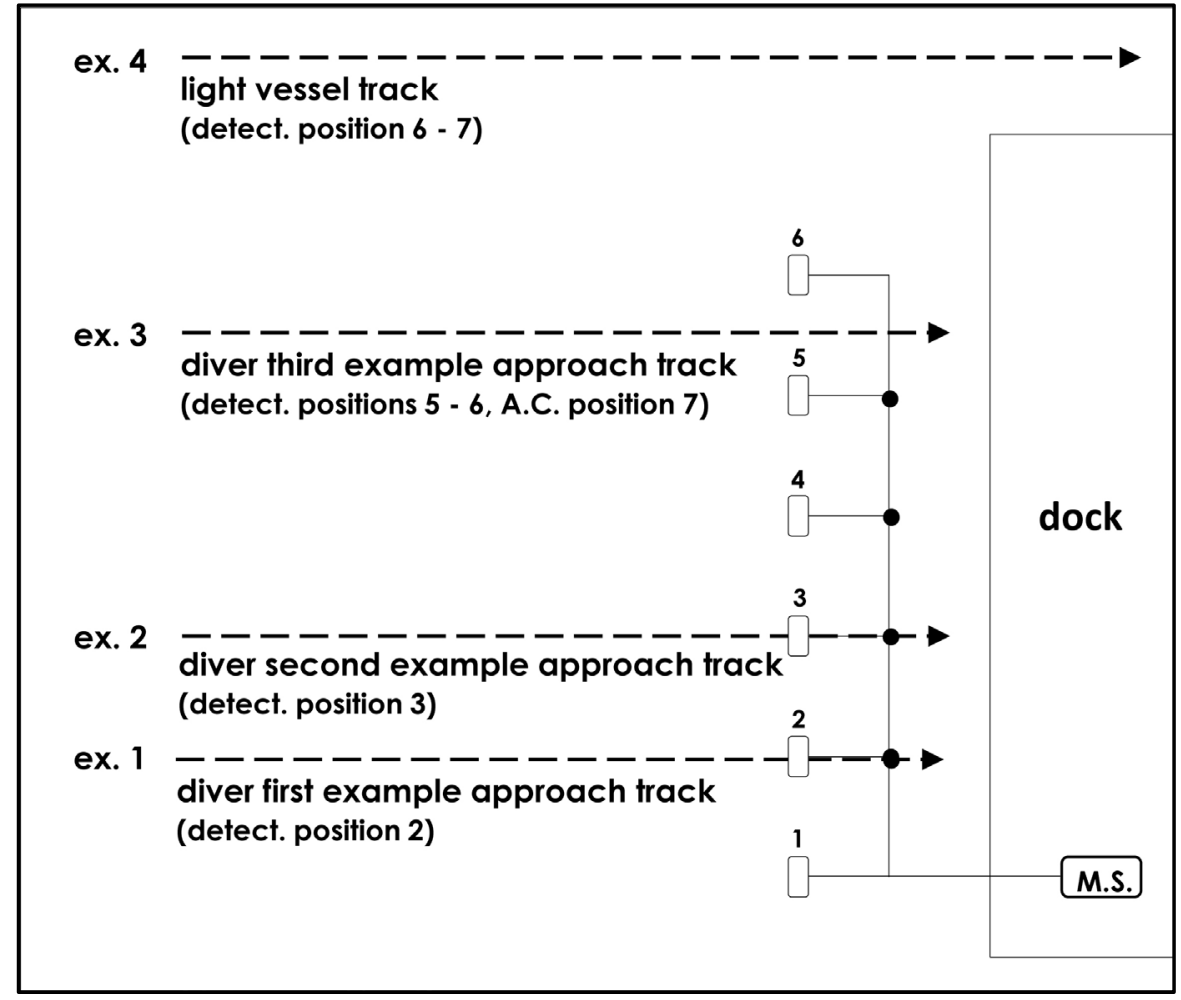

Figure 12. Picture representing the geometry of the target track and of the self-informed detection chain (qualitative). M.S. Master Station (detection and control unit). (Graphic reference for Figure 9, Figure 10 and Figure 14, Figure 16, Figure 17).

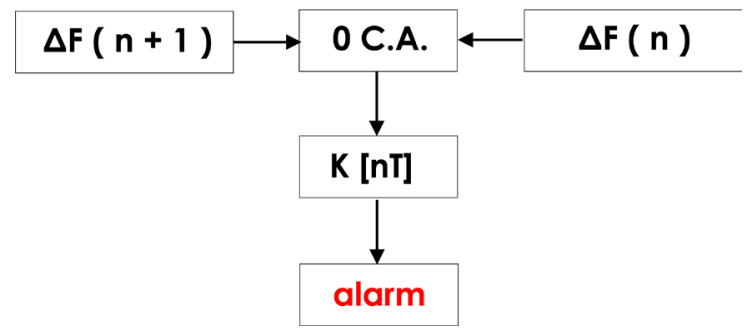

Figure 13. Zero Control Area "0 C.A." flow. The singularity conditions are classified by the comparison between the Mag $N+1$ sensor and the Mag $\mathrm{N}$ one.

In Figure 12 (ex. 2), the crossing of the detection barrier occurs above sensor 3 (detector magnetometer) and the magnetometer 2 plays as the reference one.

In the trials area, there was an oceanographic vessel conducting seafloor observation activities (instrumental tests), hence the magnetic noise observed in the reference magnetograms as per Figure 10(a) and Figure 10(b) reflected those activities.

Although the target signal was corrupted by the recurring artificial signal (Figure 10(a)) because the two signals were almost coherent in phase, the action of the self-informing protocol induced the recurring signal to be classified as noise and highlighted the signal of the target (Figure 10(c)).

If one tried to filter the recurring signal as the magnetogram in Figure 10(a) by applying standard techniques (e.g., a frequency filter), one would also filter 
out the target signal due to the similarities in the frequency band.

The metrological self-informing approach allowed to solve the singularity detection problem even when the signals were corrupted by a periodic noise (a quite frequent condition in the case of harbor fields or in presence of human activities) (Figure 10(c)).

Ambiguity Control Area (A.C.A.) performance

The self-informing protocol paragraph (Figure 8), in the architecture of the self-informed system for magnetic detection, brings about an ambiguity condition due to the symmetric geometry in the observation sensor chain and the crossing vector of the target source.

When the target halfway crosses the sensor chain between a pair of adjacent nodes (Figure 12, ex 4), its energy splits between both sensors equally (Figure 14(a) and Figure 14(b)), hence the deconvolution of the reference signal (acquired by both magnetometers) removes the informative signal recorded by both sensors (Figure 14(c)).

To overcome this ambiguity, the magnetograms of the $n-t h$ and $(n+1)$-th instruments were compared with the $(n-1)$-th one, according to the scheme in Figure 15: the $(n-1)$-th magnetometer (thus completing the self-informed elementary cell) were not affected by the singularity in the $n$-th one and the $(n+$ 1) -th one, because of its observation distance $d$ from the target $T$ :

$$
\begin{gathered}
d_{n+1 \rightarrow T} \approx d_{n \rightarrow T} \approx L / 2 \\
d_{n-1 \rightarrow T} \approx 3 L / 2
\end{gathered}
$$

where:

- $d_{i \rightarrow T}$ is the distance between the $i$-th observation node and the target $T$;

$-n+1, n$ and $n-1$ are the observation nodes (magnetometers) that compose

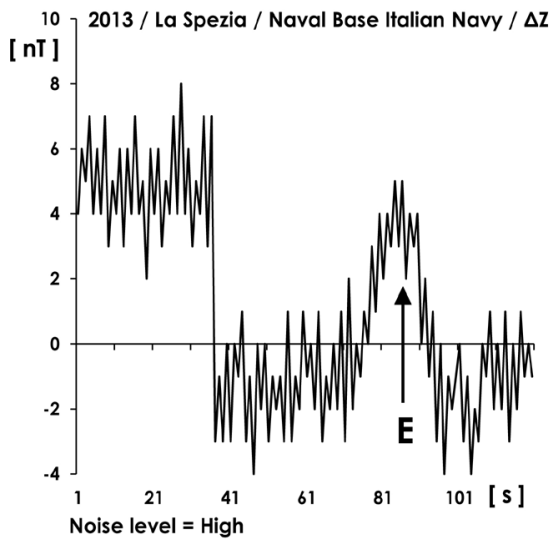

(a)

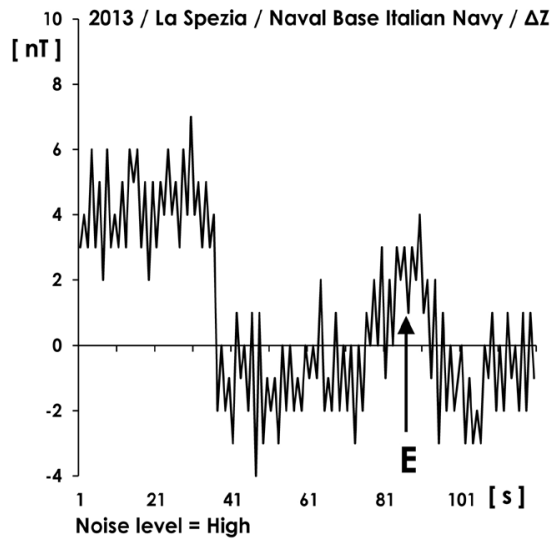

(b)

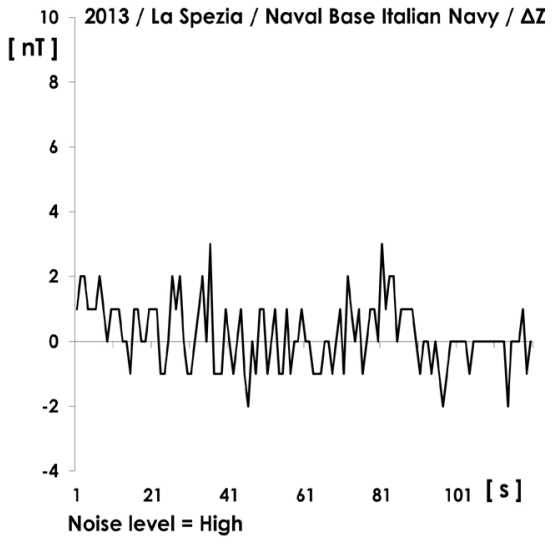

(c)

Figure 14. Example of detection ability when crossing occurs halfway between a pair of sensors. $X[\mathrm{~s}], Y$ [nT], samplig rate $f Z=1$ [Hz], $T=2$ [min], target speed: slow/medium, noise level: high, La Spezia 2013. (a) Target pulse (E) acquired by the sentinel magnetometer (mag 6) during a diver crossing halfway between the sentinel sensor and the reference one; (b) target pulse (E) acquired by the reference magnetometer (mag 5) during a diver crossing halfway between the sentinel sensor and the reference one; (c) signal coming out from the deconvolution of mag 5 recording (reference) from mag 6 recording (sentinel). The singularity related to the diver passage is removed from the deconvolution filtering because it affects both observation points (sentinel and reference) equally. 


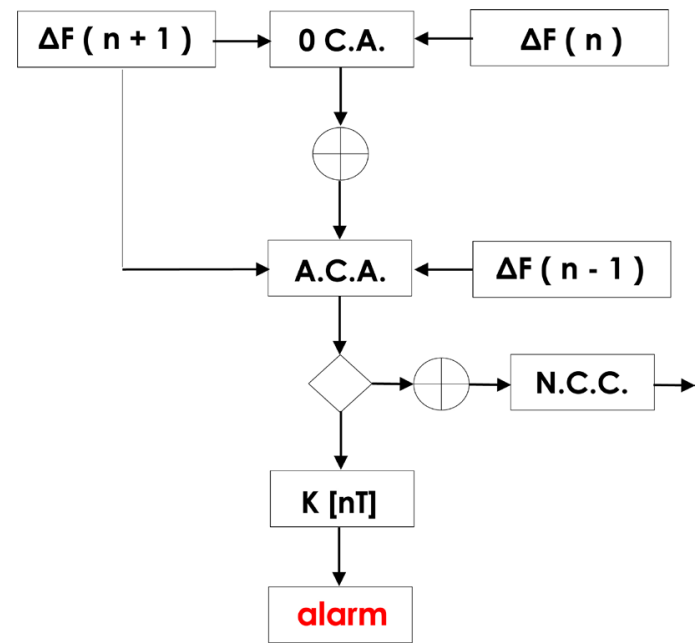

Figure 15. Ambiguity Control Area "A.C.A." flow. The absence of singularity conditions starts the control protocol to exclude halfway transit conditions, by means of a further control step referred to next sensor (grey marked steps). If singularity $=0$ go to N.C.C. $(\mathrm{New}$ Control Cicle $) \rightarrow$ if singulariry $=K[\mathrm{nT}]$ go to ALARM.

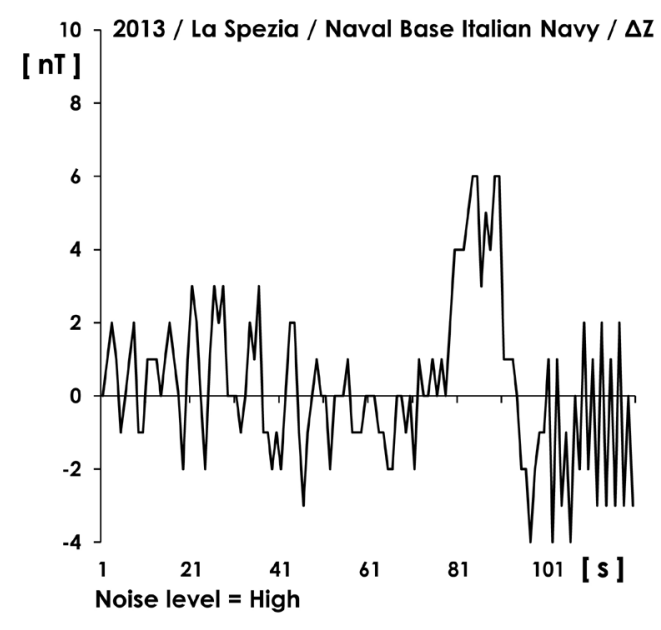

(a)

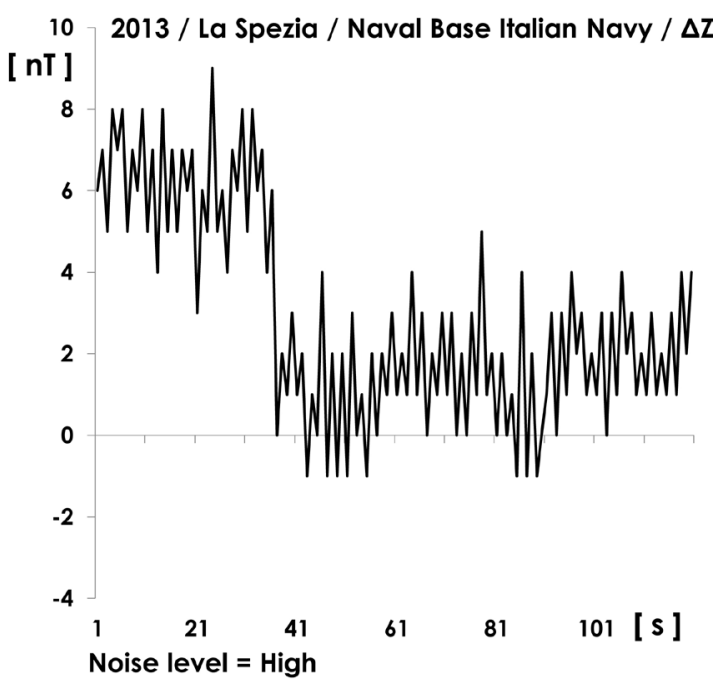

(b)

Figure 16. (a) Acquisition by the second sentinel sensor (second order singularity "A.C.A." control protocol), 2L distance from reference sensor; (b) signal coming out from the deconvolution of mag 4 recording (A.C.A. reference - second order) from the mag 6 recording (sentinel). The singularity related to the diver crossing survives the decorrelation filtering and then the system generates an alarm. $X[\mathrm{~s}], Y[\mathrm{nT}]$, samplig rate $f Z=1[\mathrm{~Hz}], T=2[\mathrm{~min}]$, v target: slow/medium, noise level: high, La Spezia 2013.

the elementary cell (Figure 16(a)).

As a result, deconvoluting the signal $F_{(n-1)}$ from the signal $F_{(n+1)}$ (or identical from the $F_{(n)}$ signal) allowed to detect the singularity as expected (Figure 16(b)).

\section{Architecture of Low Frequency Detection (Ghost Signals Cutting Procedure)}

The main operational problem concerning the stability in the detection response, is given by the entrance of an oversampled singularity in the processing 
chain of the system (a singularity signal characterized by the wavelength $\Lambda$ that is greater than the distance $L$ of the field's reading) (Figure 12, ex 4): $\Lambda>L$.

For example, consider a boat approaching the magnetic self-referred surveillance network. This problem, as anticipated in Section 2 (Figure 5), can be numerically solved using a frequency filter (FFT-HP type) that excludes these large singularities. The end user is often interested in obtaining information about the transit of this kind of sources (especially if it is uw traffic) [3].

The FFT-HP stabilization filter (Figure 5), as discuss in section 4, cannot be applied because it cuts low-frequency information out. Although one can easily recover the low-frequency information excluded from the FFT-HP filter and deploy a specific computing channel dedicated to this component, the metrological problem of the instability in SUPERGUARD at low-frequency responses is not overcome (Figure 17). This occurs because the displacement of the source with respect to the observation chain generates a phase shift in the signal recordings between the observation magnetometers with respect to the source speed:

$$
\Phi=\Phi_{v}
$$

If one adopts as reference signal the trace shown in Figure 18 (mag 6 - Figure 12 ), the comparison of the signal detected from the nearest magnetometer (mag 5 - Figure 12) with the reference one (Figure 19) generates a residue of dipolar computation (ghost signal) (Figure 20): the distance $L$ between the two observation points is not sufficient to decorrelate the singularity ( $\Lambda$ of the oversampled singularity).

The ghost signal is a numerical artifact and makes no physical sense, hence the generated alarm would be a typical False Alarm, thus lowering the confidence in the system responses. Such a phenomenon persists even if one considers a farther magnetometer as a reference for the deconvolution's routine (e.g.

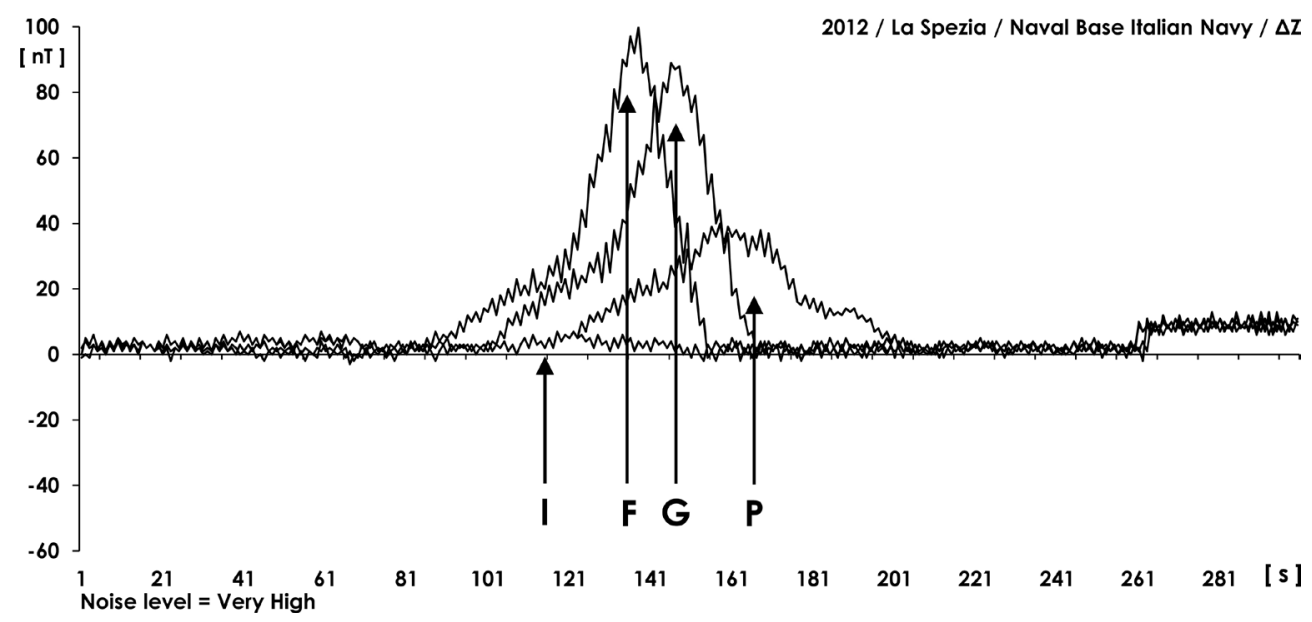

Figure 17. F - G - P: example of singularities family coming from an oversampled signal $(\Lambda$ target signal $\gg L$ ) - sensors $6,5,4$. The I acquisition comes from a far reference magnetometer (in this present case located $\mathrm{H}=13 \mathrm{~L}$ distance from sentinel magnetometer). $X[\mathrm{~s}], Y[\mathrm{nT}]$, samplig rate $f Z=1$ [Hz], $T=5$ [min], v target: slow/medium, noise level: very high, La Spezia 2012. 


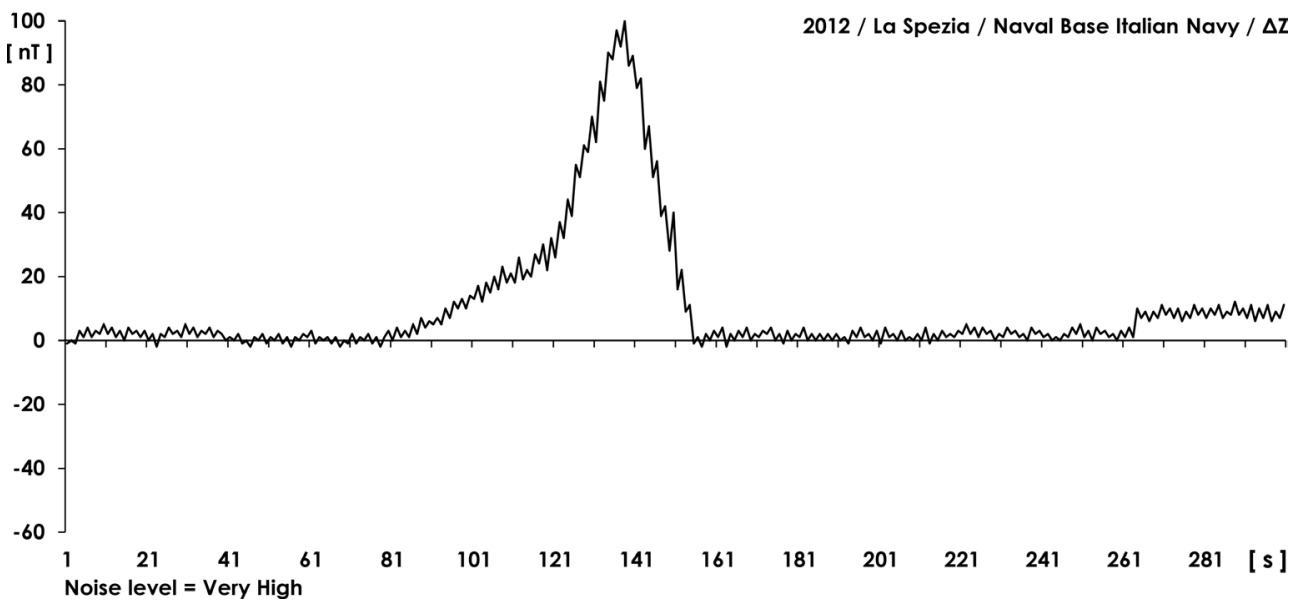

Figure 18. Large wavelength $(\Lambda)$ singularity (see F pulse in Figure 17) induced in mag 6 recording by a light vessel moving near the sensor chain along a perpendicular track. $X[\mathrm{~s}], Y[\mathrm{nT}]$, samplig rate $f Z=1[\mathrm{~Hz}], T=5[\mathrm{~min}]$, v target: slow/medium, noise level: very high, La Spezia 2012.

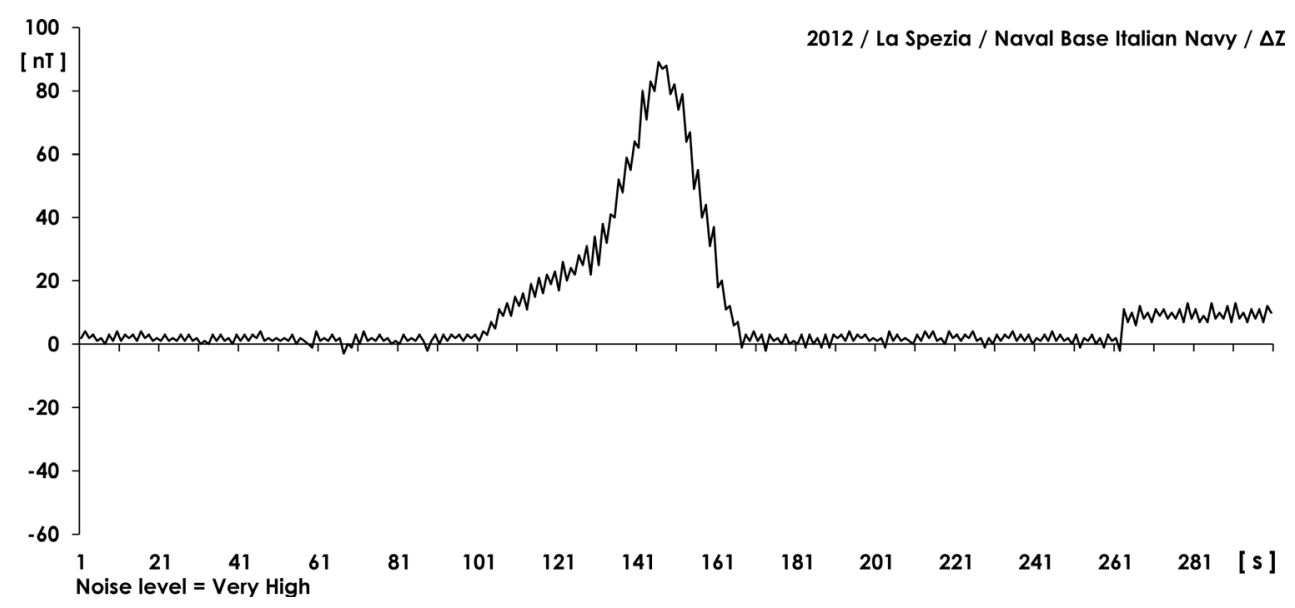

Figure 19. Time migration (and amplitude decreasing) of the singularity $\mathrm{G}$ (see Figure 17) in mag 5 recording; distance mag $5-\operatorname{mag} 6=L . X[\mathrm{~s}], Y[\mathrm{nT}]$, samplig rate $f z=1[\mathrm{~Hz}], T=5[\mathrm{~min}], \mathrm{v} \operatorname{tar}-$ get: slow/medium, noise level: very high, La Spezia 2012.

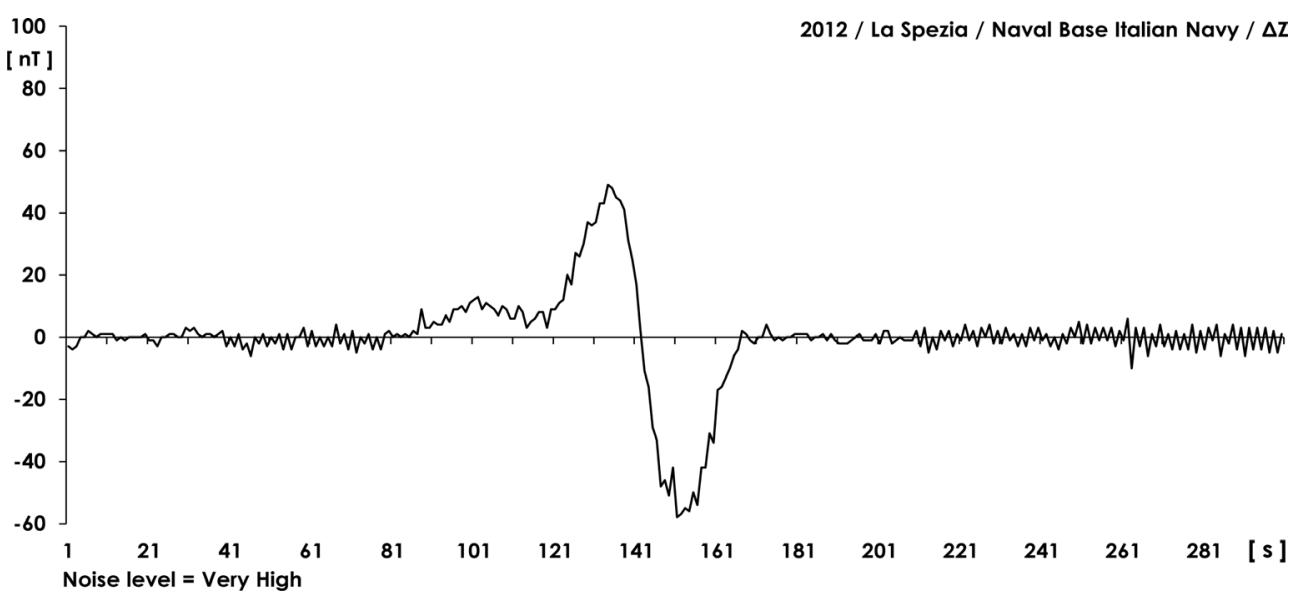

Figure 20. Signal coming out from the deconvolution of $G \rightarrow F$ (Figure 17). This signal has not physical meaning but is a computation residue due to the phase shift of the reference signal compared to the measurement one (F-mag 6). $\Lambda \gg L(\mathrm{G}-$ mag 5$)$. 
mag 3 - Figure 12), because even the $3 L$ distance is not sufficient to decorrelate the signal from the noise (Figure 21).

In fact, even though the signal in Figure 21 was characterized by a very low amplitude (Figure 18), the phase shift between the two signals generated a ghost signal having amplitude similar to that in Figure 20, and characterized by a greater wavelength which depends on the phase shift $\Phi$. (Figure 22)

$$
\Lambda(g s)=\Lambda(g s)_{\Phi}
$$

This mechanism could provide interesting information about the source speed but not about the physical consistency of the differential detection signal produced by SUPERGUARD.

The problem can be overcome by setting the reference node far enough from the measurement one (Figure 23). The signal measured by such a reference

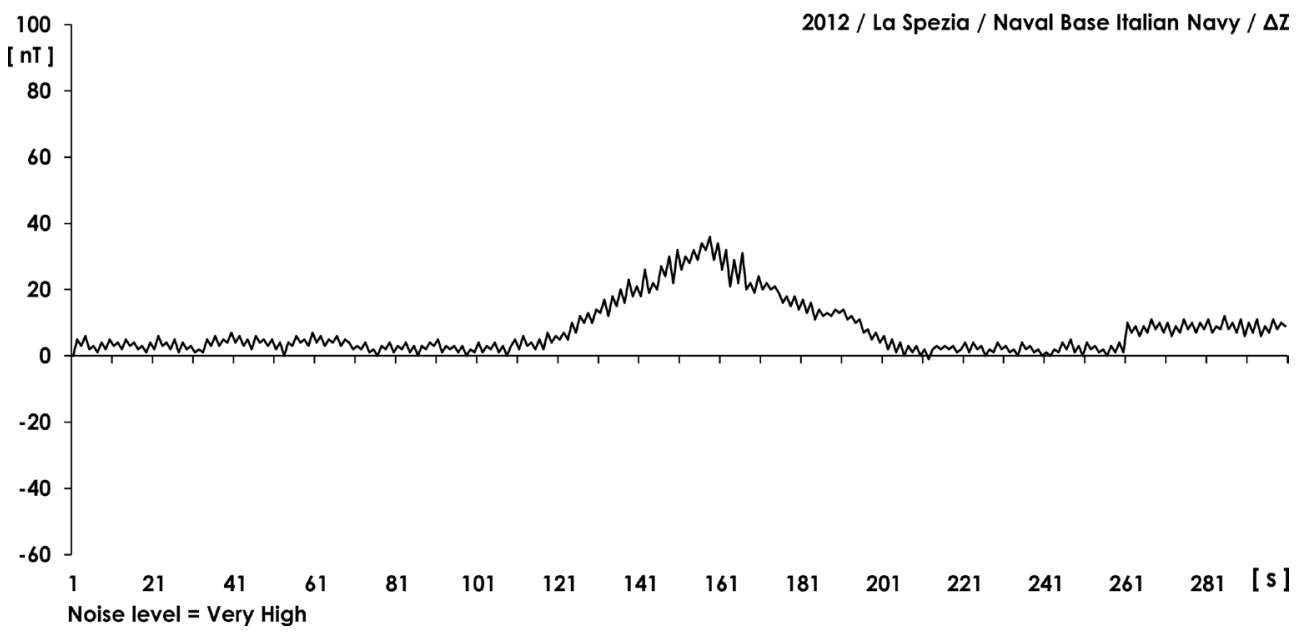

Figure 21. Time migration (and amplitude decreasing) of the singularity $\mathrm{P}$ (see Figure 17) in mag 3 recording; distance mag 3 - mag $6=3 L . X[\mathrm{~s}], Y[\mathrm{nT}]$, samplig rate $f Z=1[\mathrm{~Hz}], T=5[\mathrm{~min}]$, v target: slow/medium, noise level: very high, La Spezia 2012.

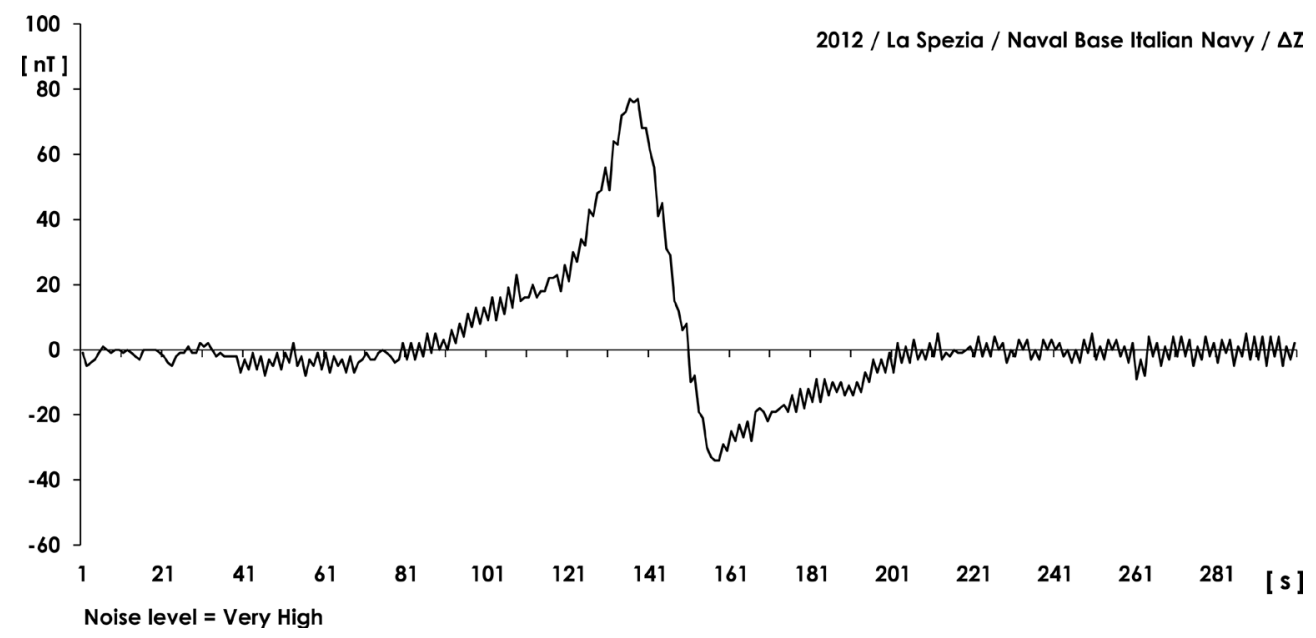

Figure 22. Signal coming out from the deconvolution of $P \rightarrow F$ (Figure 17). This signal has not physical meaning but is a computation residue due to the phase shift of the reference signal compared to the measurement one (F-mag 6). $\Lambda \gg L(\mathrm{P}-\operatorname{mag} 3)$. 


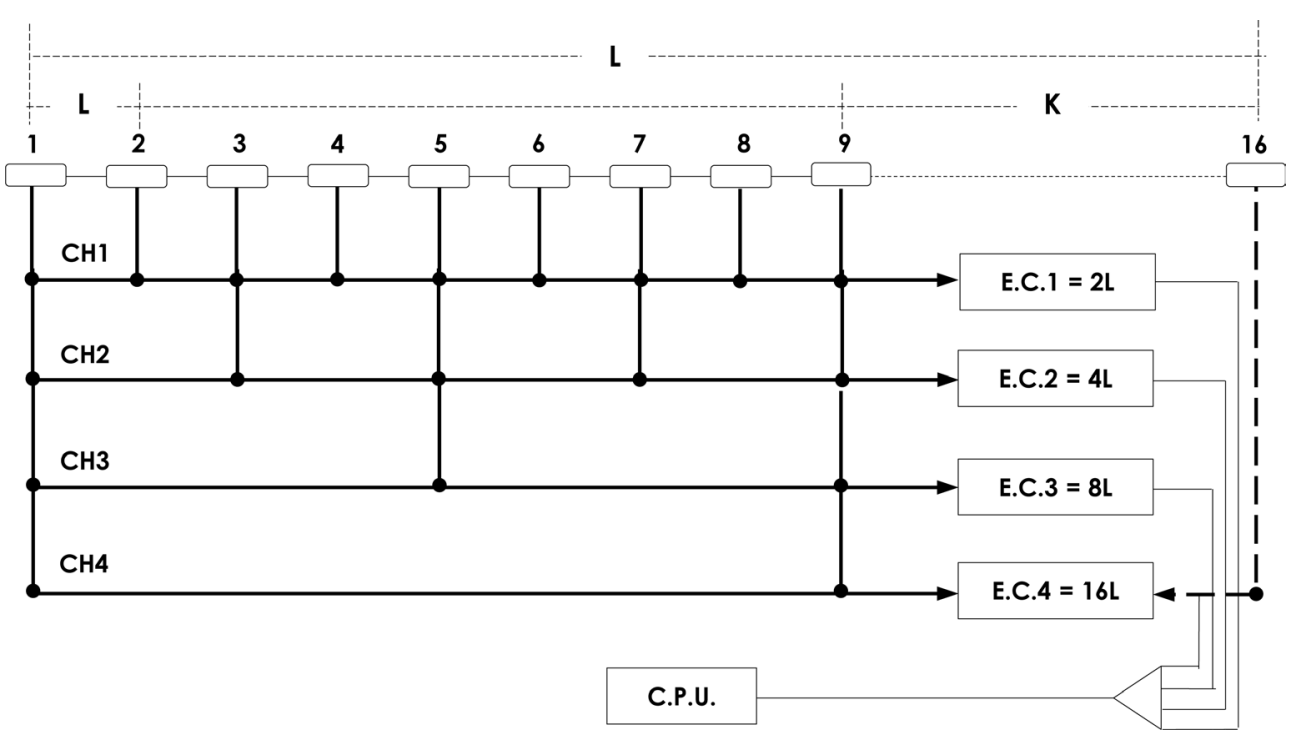

Figure 23. Extension of the elementary cell length to detect increasing wavelength signals. (Mags 1 - 2 to compute wavength signals $\cong 2 L$ (channel 1 - CH1); Mags 1-3 $2 L<\Lambda \leq 4 L$ (channel 2 CH2); ...; Mags $1-168 L<\Lambda \leq L$ ).

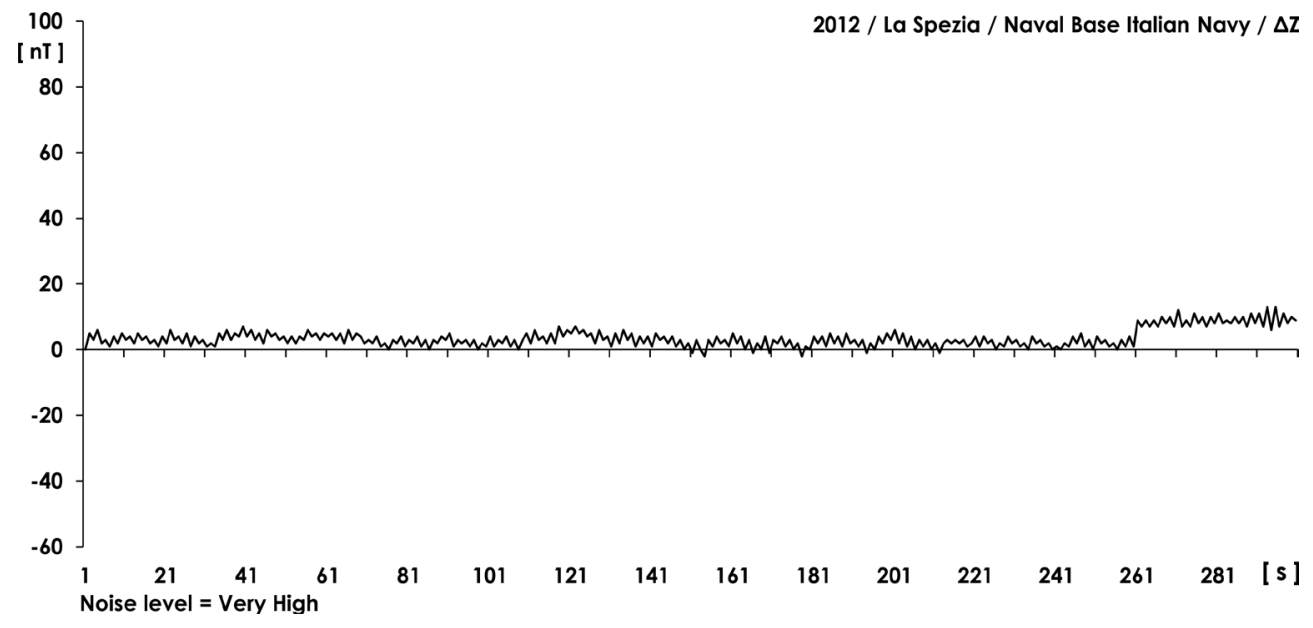

Figure 24. Signal (I) of the field measured at decorrelation distance between the sentinel magnetometer and the reference one (distance between the sensors $H \gg L$ ); the signal is not influenced by the singularity. $X[\mathrm{~s}], Y[\mathrm{nT}]$, samplig rate $f z=1[\mathrm{~Hz}], T=5[\mathrm{~min}], V$ target: slow/medium, noise level: very high, La Spezia 2012.

node (in our case, a reference magnetometer was placed at a distance equal to 13 $L$ ) is free from the corruption due to the singularity (Figure 24) and therefore its decorrelation from the signal acquired by the sentinel magnetometer provide a real response, hence the alarm is valid (Figure 25).

The solution proposed here is therefore to perform a series of deconvolution comparisons at variable distances: first, a comparison between magnetometers 1 and 2 using magnetometer 3 as a reference; secondly, a comparison between magnetometers 1 and 3, using magnetometer 5 as a reference; next, $n$-order comparison between magnetometers 1 and $n+1$, using magnetometer $2 n+1$ as reference (Figure 23): 


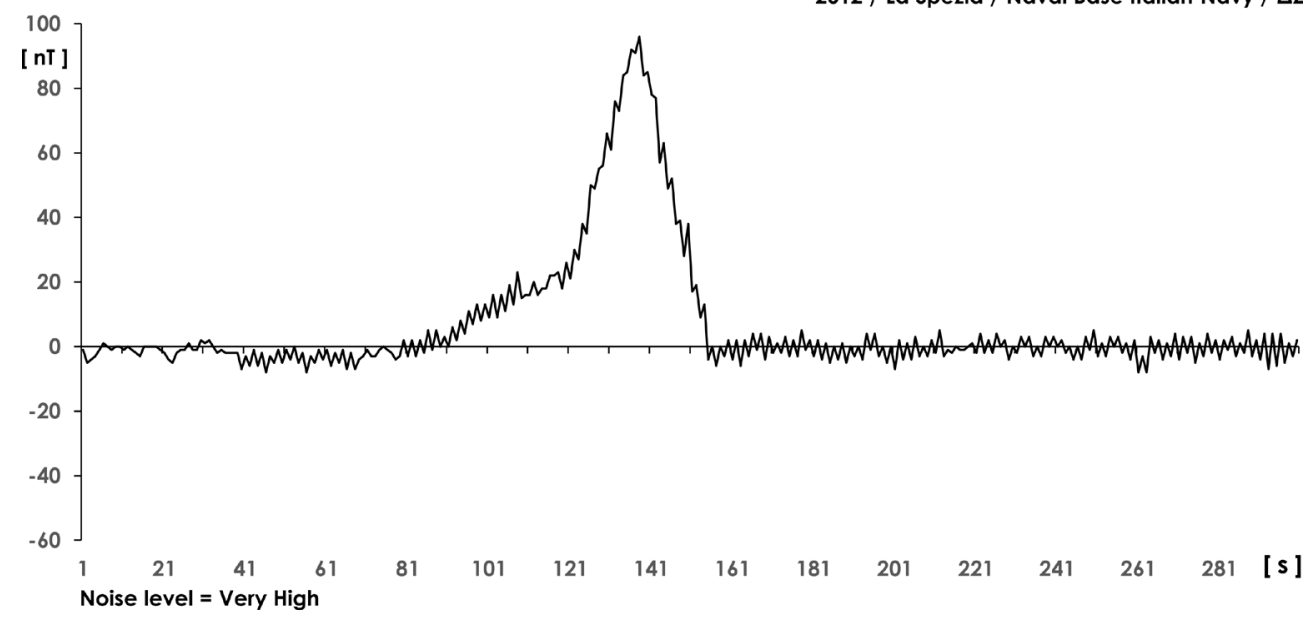

Figure 25. Signal coming out from the deconvolution of $\mathrm{I} \rightarrow \mathrm{F}$ (Figure 17). This signal has physical meaning because the distance between the reference magnetometer $(H=13 L)$ is sufficient to decorrelate the target signal (even if this has large wavelength). The system alarms.

Table 2. Statistic of the effectivness in the singularity detection. column 1: umber of detections; column 2: reference organization for the experiments; column 3: their nationality; column 4: location of the experiment; column 5: real detection; column 6: detection uncertainity; column 7: false detections.

\begin{tabular}{ccccccc}
\hline N. Signals & Command & Navy & Port of Exp. & R & U & F \\
\hline 231 & MARI.CO.DRAG. & ITA & La Spezia (ITA) & 228 & 2 & 1 \\
22 & COM.SUB.IN. & ITA & La Spezia (ITA) & 22 & - & - \\
27 & WTD.71 & GER & Eckemförde (GER) & 27 & - & - \\
\hline
\end{tabular}

$$
H \gg L
$$

\section{Experimental Overview}

During the study and testing period of the system for detecting underwater magnetic singularities in ionic low-density solutions (2008 - today), over two hundreds samples of signal were recorded, generated by divers in simulation of attacks to sensible terrestrial plants in noisy geomagnetic harbor UW environments (Table 2).

Measurements and validation operations were performed by military operators during official research programs.

The SUPERGUARD system scored 274 alarms out of a total of 277 recorded singularity signals, hence the error rate barely exceeded $1 \%$.

The same system of sensors, in the same measurement locations, under the same magnetic environmental noise conditions, used in the conventional way (direct measure + techniques of signal robustness in FFT) gave a Detection probability between $15 \%$ and $38 \%$ with a False Alarm rate always greater than $45 \%$.

\section{Conclusions}

The magnetic singularity detection procedure (based on the self-information 
concept) was tested in several projects supported at a national level by the "Piano Nazionale Ricerche Militari" (Ministry of Defence - Italy) and, at the European level, by the European Defence Agency.

The project focused on the study of detection effectiveness of the variations of $Z$ component $(\Delta Z)$ of the magnetic field $F$.

The technique allowed to shift the geomagnetic field metrology from a directmeasurement approach (the measure of the field by magnetometers) to a singularity-measurement one (differential field measure based on the internodal distance $L$ to correlate the noise and decorrelate the target signal). As a result metrology associated to instrumental sensitivity evolved toward system sensitivity.

The results obtained confirm the system effectiveness in acquiring and correctly describing the singularity (anomaly) associated with underwater sources, especially when the latter generated weak signals, due to their quasi-punctiform and kinematic nature (e.g. diver in seawater).

At present the "SUPERGUARD" system is an operational prototype including:

1) Hardware components (commercial magneto variometers, standard communication systems and a standard PC as management and control station);

2) Software numerical self-informing protocols installed on a PC.

The software self-informing protocols are ready to be implemented in hardware circuitry.

As a result of the four-year campaign in high-definition EMAG underwater metrology, we consider technically feasible the detection of divers in an underwater peripheral marine environment, also in highly noisy conditions such as harbors.

\section{Acknowledgements}

This study is part of the Italian Harbor Protection research action; it was supported by SEGREDIFESA of the Italian Ministry of Defence under the National Military Research Plan R\&T project La.Ma. and EDA (European Defence Agency) project Ha.P.S. (SWE Lead Nation, ITA, GER, NOR).

Command C.S.S.N. (Italian Navy) managed the research (La.Ma.-1.0/2.0 Projects), Commands MARI.CO.DRAG, COM.SUB.IN. (Italian Navy) and WTD71 (Bundeswehr) supported the field experiments (La.Ma.-1.0/2.0 and HaPS Projects and six other trials of system effectiveness evaluation). The technological support of SkyTech Company (SP - ITA) was invaluable for our work.

These results would not have been achieved without the appreciation and encouragement of Admirals Salvatore Teja and Massimo Marchesi (C.S.S.N. - Italian Navy).

Comments and suggestions of JSIP anonymous reviewers improved the paper.

\section{References}

[1] Harris, F. (1978) On the Use of Windows for Harmonic Analysis with the Discrete Fourier Transform. Proceedings of IEEE, 66, 51-83. 
https://doi.org/10.1109/PROC.1978.10837

[2] Faggioni, O., Soldani, M., Leoncini, D.A., Crise, A., Grandinetti, P., Nasta, S. and Zunino, R. (2012) A New EMAG Metrological Approach for Submarine Detection: Fundamentals of the Numerical Protocols and Preliminary Field Performance. Proceedings of the 25th UDT Undersea Defence Technology Europe Conference, Ifa-Alicante, 29-31 May 2012, CD-ROM.

[3] Gabellone, A., Faggioni, O., Soldani, M. and Guerrini, P. (2007) C.A.I.Ma.N. Experiment. Proceedings of the 20th UDT Undersea Defence Technology Europe Conference, Naples, 5-7 June 2007, CD-ROM.

[4] Faggioni, O., Pinna, E., Savelli, C. and Schreider, A.A. (1995) Geomagnetism and Age Study of Tyrrhenian Seamounts. Geophysical Journal International, 123, 915-930. https://doi.org/10.1111/j.1365-246X.1995.tb06898.x

[5] Rikitake, T. (1958) Oscillations of a System of Disk Dynamos. Mathematical Proceedings of the Cambridge Philosophical Society, 54, 89-105. https://doi.org/10.1017/S0305004100033223

[6] De Vuyst, A. and De Meyer, F. (1973) Spectral Analysis of Geomagnetic Data from One Station (Dourbes 1960-1970). Institut Royal Meteorologique de Belgique, Publ., A, No. 80, 32.

[7] Menvielle, M. and Berthelier, A. (1991) The K-Derived Planetary Indices: Description and Availability. Reviews of Geophysics, 29, 415-432.

https://doi.org/10.1029/91RG00994

[8] Faggioni, O., Soldani, M., Gabellone, A., Hollett, R.D. and Kessel, R.T. (2010) Undersea Harbour Defence: A New Choice in Magnetic Networks. Journal of Applied Geophysics, 72, 46-56. https://doi.org/10.1016/j.jappgeo.2010.07.001

[9] De Santis, A. (1993) Tempeste, Sottotempeste e baie magnetiche. Annali di Geofisica, 36, 55-77.

[10] NOAA, et al. (2010) NOAA Diving Manual: Diving for Science and Technology. 4th Edition Revised, Best Publishing Company, North Palm Beach.

[11] COMSUBIN (2011) Norme per le Immersioni. Marina Militare, Roma.

[12] Decherchi, S., Leoncini, D., Gastaldo, P., Zunino, R., Faggioni, O. and Soldani, M. (2011) Computational Intelligence Methods for Underwater Magnetic-Based Protection Systems. Proceedings of IJCNN International Joint Conference on Neural Networks, San Jose, 31 July-5 August 2011, CD-ROM.

[13] Leoncini, D., Decherchi, S., Faggioni, O., Gastaldo, P., Soldani, M. and Zunino, R. (2010) Linear SVM for Underwater Magnetic Signals Based Port Protection. Journal of Information Assurance and Security, 5, 401-408.

[14] Gabellone, A., Faggioni, O., Soldani, M. and Guerrini, P. (2008) C.A.I.ma.N. Coastal Anti Intruder Magnetometers Network. Proceedings of RTO-MP-SET-130 Symposium on NATO military sensing, Orlando, 12-14 March 2008, CD-ROM.

[15] Faggioni, O., Soldani, M., Leoncini, D., Gabellone, A. and Maggiani, P.V. (2009) Time Domain Performances Analysis of Underwater Magnetic SIMAN Systems for Port Protection. Journal of Information Assurance and Security, 4, 538-545.

[16] Ramirez, R.W. (1985) The FFT: Fundamentals and Concepts. Prentice-Hall, Upper Saddle River.

[17] Kanasewich, E.R. (1973) Time Sequence Analysis in Geophysics. The Alberta University Press, Edmonton. 